Effects of Spectral Truncation on General Circulation and LongRange Prediction

\author{
By \\ F. Baer and F.N. Alyea
}

Department of Atmospheric Science

Colorado State University

Fort Collins, Colorado

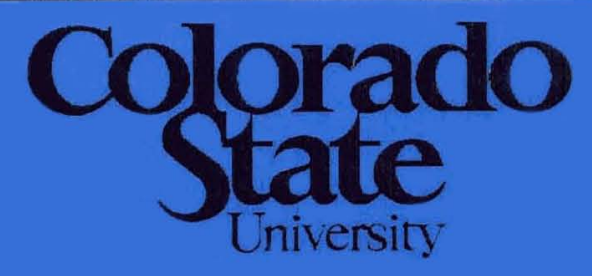

Department of
Atmospheric Science

Paper No. 169 
Reprinted from Jurrial of Ammospifrre Sctexces, Vol. 28, No. 4. May, 1971, pp. 457-480 American Meteorological Society P'rinted in U.S. $A$.

\title{
Effects of Spectral Truncation on General Circulation and Long-Range Prediction ${ }^{1}$
}

\author{
F. Baer and F. N. Alyea \\ Depl. of Almospheric Science, Colorado Slate Unicersily, Fi. Collins
}

(Manuscript received 15 January 1971)

\begin{abstract}
Although atmospheric prediction models appear to yield results similar to observation, both their detailed predictive capalility and their time-averaged forecasts depend on space truncation. Such dependence may be readily studied with a spectral model because of the ease of modifying truncation. A simple, two-level, quasi-geostrophic, forced general circulation model was represented in sjectral form and nine cases of different truncation were integrated for the same forcing, starting with initial conditions generated from a state of rest. The truncations ranged from six to sixteen meridional waves, from five to ten degrees of freedom with latitude, and the models were integrated for about 60 days with finite-amplitude nonlinearity. Considering the kinetic energy in the vertical mean flow, and separating this energy into zonal and eddy, the results show that the general circulation may be predicted with as few as twelve planetary waves and eight latitudinal degrees of freedom, whereas detailed prediction for a period of 15-20 days requires at least sixteen planetary waves and eight to ten latitudinal degrees of freedom. The broad variation in solutions for different truncations observed in this study implies that care must be taken in selecting space truncation for any physical model chosen for integration.
\end{abstract}

\section{Introduction}

The ultimate solution to the problem of large-scale numerical weather prediction, if indeed a unique solution exists, will rest on the thorough understanding of several factors arising principally from the fact that the problem is basically nonlinear. These factors include a proper analytic specitication of the physical forces and accelerations, the influence of initial conditions on the solutions as a function of time, and the impact of truncation (computational procedures) as the solution proceeds with time. The latter thorn arises from the lack of exact solutions to complex nonlinear systems thereby requiring numerical integration of a non-continuous (truncated) form of the exact system.

Significant progress has been made in the development of models which include detailed specification of physical processes, and further refinements will undoubtedly be forthcoming as more information about the atmosphere becomes available. These models have furthermore been specifically designed to consider different integration periods, such as short-range prediction ( $\sim 3$ days), long-range prediction ( $\sim 3$ weeks), and the general circulation. Investigations into the impact of initial conditions go on apace. Since the literature is replete with results of such studies, specific references will be neglected.

${ }^{1}$ Presented at the AMS-IMS International Conference on Meteorology, Israel, 30 November-4 December 1970. ('omputational approximations and their impact on solutions have been studied traclitionally by application to linear systems, a procedure validated by allowing comparison to known, exact solutions. A considerable body of knowledge exists in this area, but unfortunately the same cannot be said for nonlinear problems as exemplified by the atmospheric prediction problem. Moreover, this problem is compounded by requiring truncation in both space and time. Although experimenters in testing nonlinear models utilize what information is available to maintain computational stability in their integrations (and this basically implies the extrapolation of information from linearized systems), little systematic study has been applied to the effect on solutions of variations in truncation, especially space truncation, with all other factors held fixed.

With the intent to increase our understanding of the influence of space truncation in numerical weather prediction, we have chosen a simple, but hopefully meaningful, physical model incorporating in a crude way the basic physics as it applies to motions in the atmosphere. The model contains two atmospheric layers with fixedstability, internal and surface stresses, and an inhomogeneous heat source to account for energy input. It will become apparent from the text that the incorporation of a forcing function obviates the need to specify initial conditions, thereby by passing one of the aforementioned unresolved problems in numerical weather prediction. We have integrated this model numerically in time for 
a number of different truncations (in all cases using the same time extrapolation procedure), starting with a self-generated zonal flow distribution by which wave activity was amplified.

To further simplify the task of varying truncation for different experiments," we have represented our model equations in the "spectral" domain, wherein the time-space dependence of dependent variables in a horizontal surface is characterized by a series of unknown time-dependent coefficients multiplied by known orthogonal polynomials (in this case Legendre polynomials). The entire system may then be written as a set of ordinary nonlinear differential equations in time for the unknown coefficients. Space truncation, defined in terms of this representation as "spectral" truncation, is thus simply established by the point at which the series expansion is terminated. We have chosen nine different truncations (truncation points) and discuss the results of the consequent integrations with reference to them. The reader may easily re-interpret the results in terms of truncation in the finite-difference domain.

The results of these integrations will be interpreted from the viewpoint that beyond a certain level of resolution (which may not have been reached by any of our truncations) all experiments will yickl similar, if not identical, solutions. To assess the results of any experiment we shall depict primarily the behavior of the cnergy in the system and its variation in time; no effort has been made to consider phase changes. Furthermore, since there is a significant difference in prediction requirements for long-range prediction and the general circulation, we have studied the effects of truncation for both these problems. Finally, influences due to truncation may be assessed independently of the fidelity of computational results to observed atmospheric events; the reader is consequently alerted to avoid interpreting the results in terms of their "correctness" but rather in terms of truncation.

\section{The model}

To establish the influence of space truncation on the ultimate prediction of an atmospheric model, a gencral circulation model would be most appropriate because it bypasses the influence of initial conditions. Many such models of varying sophistication have been developed in recent years (see, for example, Smagorinsky et al., 1965; Kasahara and Washington, 1967; Leith, 1965; and Mintz, 1965). The problem of truncation may best be studied with a simpler model-both to

\footnotetext{
${ }^{2}$ This term is used synonymously in the text with numerical integrations or calculations since they refer to the results for a given truncation.
}

avoid complexity in the physics and to limit computing requirements - but one which incorporates real atmospheric processes sufficiently to yield realistic predictions. Such a model was first presented by Phillips (1956) and later by Charney (1959). We will utilize this model as represented in an energetically consistent fashion by Lorenz (1960).

Our model utilizes the quasi-geostrophic assumption, is quasi-hydrostatic, has fixed static stability, and incorporates horizontal diffusion, vertical shearing st resses both internally and at the lower boundary, and a forcing function to describe heating. Represented in pressure $(p)$ coordinates, the levels $1000,750,500,250$ and $0 \mathrm{mb}$ are referred to by the subscripts $0-4$, respectively. The vertical pressure velocity vanishes at both top and bottom of the model atmosphere, i.e., $\omega_{0}=\omega_{1}=0$. The horizontal wind field (V) may be represented in terms of a streamfunction $(\psi)$ where we have $V=k \times \Gamma \psi$, with $\mathbf{k}$ describing the unit vector in the direction of increasing geopotential (\$). Following Lorenz (1900), we may describe both the stream ficld and the thermal field in terms of a mean and shear as follows:

$$
\left.\begin{array}{l}
\psi=\frac{\psi_{1}+\psi_{3}}{2} \\
\tau=\frac{\psi_{3}-\psi_{1}}{2} \\
\theta=\frac{\theta_{3}+\theta_{1}}{2} \\
\sigma=-\frac{\theta_{3}-\theta_{1}}{2}
\end{array}\right\},
$$

where $\theta$ is potential temperature, and $\sigma$ static stability; a constant. Geostrophically, moreover, the mean potential temperature is related to the stream shear by

$$
\theta=\frac{f_{0}}{b c_{p}} \tau+\text { constant }
$$

where $f_{0}$ is a mean Coriolis parameter and $b=0.124$.

The heating function (to be discussed in more detail subsecquently) is defined as

$$
H=\frac{1}{c_{p}}-2^{\times} \dot{Q},
$$

and the vertical component of the curl of stress and diffusion is represented by the symbol $D$.

Utilizing the above notation and approximations, we arrive at the dynamic equations for this model in 
the following form (see Lorenz, 1960):

$$
\begin{aligned}
& \frac{\partial \theta}{\partial t}=-J(\psi, \theta)+\frac{2 \sigma}{p_{0}} \omega_{2}+I I \\
& \partial \\
& \Gamma^{2} \psi=-J\left(\psi, \nabla^{2} \psi+f\right)-J\left(\tau, \Gamma^{2} \tau\right) \\
& \frac{\partial}{\partial t} \nabla^{2} \tau=-J\left(\psi, \Gamma^{2} r\right)-J\left(\tau, \nabla^{2} \psi+f\right) \\
& +\frac{D_{3}-D_{1}}{2}+\frac{2 \rho_{0}}{p_{0}} \omega_{2}
\end{aligned}
$$

In (3), I refers to the Jacobian in the coordinates on the spherical surface, i.e.,

$$
J(\alpha, \beta)=\frac{\partial(\alpha, \beta)}{a^{2} \partial(\lambda, \mu)}
$$

where $\lambda$ represents longitude, $\phi$ latitude, and $\mu=\sin \phi$. Utilization of the geostrophic condition (2) and simullaneous solution of the first and third of Eqs. (3) will yield two predictive equations in $\psi$ and $\tau$.

let us consider now the forms of diffusion and stress. We define the dissipation forces as

$$
\mathbf{F}=A \nabla^{2} \mathbf{V}+g \frac{\partial \tau}{\partial p}
$$

and the contribution to the vertical component of vorticity equation as $\mathbf{k} \cdot \nabla \times \mathbf{F}$. The Laplacian operator in (A) is in the horizontal (spherical) surface, $A$ the coefficient of horizontal momentum diffusion, and $g$ the acceleration of gravity. Development in spherical coordinales $(\lambda, \phi)$ shows that

$$
\mathbf{k} \cdot \nabla \times \nabla^{2} \mathbf{V}=\nabla^{4} \psi+\frac{2 \nabla^{2} \psi}{a^{2}}-\frac{2 g \rho}{a} \frac{\partial \nabla^{2} \psi}{\partial p}
$$

where $\rho$ is the density and $a$ represents the mean radius of the earth. We ignore the last term on the right-hand side of (5) since we shall see that it is overwhelmed by the shearing stresses. Assuming that the stress at the top of the model vanishes $\left(\tau_{+}=0\right)$, we find that the intluence of stress is evaluated at the mid-level and at the surface from the conditions

$$
\left.\left.\frac{\partial \tau}{\partial p}\right)_{3}=-\frac{\tau_{2}}{\Delta p}, \frac{\partial \tau}{\partial p}\right)_{1}=\frac{\tau_{2}}{\Delta p}-\frac{\tau_{0}}{\Delta p}
$$

In the free atmosphere we choose the stress proportional to the vector wind shear whereas at the surface it is chosen proportional to the square of the wind, although we linearize for ease of computation. 'These conditions vield

$$
\left.\begin{array}{l}
\left.\tau_{2}=k_{1} \frac{\partial \mathbf{V}}{\partial p}\right)_{2}=\frac{k_{1}}{\Delta p}\left(\mathbf{V}_{3}-\mathbf{V}_{1}\right) \\
\tau_{0}=k_{c} \rho_{01}\left|\overline{\mathbf{V}}_{0}\right| \mathbf{V}_{0}=\frac{k_{c} \rho_{0}}{2}\left|\overline{\mathbf{V}}_{0}\right|\left(3 \mathbf{V}_{1}-\mathbf{V}_{3}\right)
\end{array}\right\},
$$

where the surface wind is linearly extrapolated from levels 1 and 3 . Using (6), (7), (1), and the relationship between wind and the streamfunction, we find for the contribution to vorticity change from stress in levels 1 and 3

$$
\left.\begin{array}{l}
\left.g \mathbf{k} \cdot \nabla \times \frac{\partial \tau}{\partial p}\right)_{1}=2 c_{1} \nabla^{2} \tau-2 c_{2}\left(\Gamma^{2} \psi-2 \nabla^{2} \tau\right) \\
\left.g \mathbf{k} \cdot \nabla \times \frac{\partial \tau}{\partial p}\right)_{3}=-2 c_{1} \Gamma^{2} \tau
\end{array}\right\},
$$

where $c_{1} \equiv g k_{1} / \Delta p^{2}$ and $c_{2} \equiv g k_{r} \rho_{0}\left|\overline{\mathbf{V}}_{0 \mid}\right| / 2 \Delta p$.

If we now evaluate (5) at levels 1 and 3 and combine these results with (8), the contributions of diffusion and stress to the equations for the time change of the mean and shear stream fields become

$$
\left.\begin{array}{l}
\frac{D_{3}+D_{1}}{2}=.1 \nabla^{4} \psi+c_{3} \nabla^{2} \psi+2 c_{2} \nabla^{2} \tau \\
\frac{D_{3}-D_{1}}{2}=A \nabla^{4} \tau+c_{4} \nabla^{2} \tau+c_{2} \nabla^{2} \psi
\end{array}\right\},
$$

with the definitions $c_{3} \equiv 2\left(1 / a^{2}\right)-c_{2}$ and $c_{1} \equiv 2\left[\left(1 / a^{2}\right)\right.$ $\left.-c_{1}-c_{2}\right]$. Substitution of (9) inlo (3) together with (2) will now provide us with two prediction equations in the variables $\psi$ and $\tau$. Finally, when we nondimensionalize all variables in time by the earth's rotation rate $(\Omega)$ and the earth's mean radius $(a)$, we arrive at the nondimensional prediction equations

$$
\left.\begin{array}{l}
\frac{\partial}{\partial t} \nabla^{2} \psi+L_{1} \psi-2 c_{2}^{\prime} \nabla^{2} \tau=-J\left(\psi, \nabla^{2} \psi\right)-J\left(\tau, \Gamma^{2} \tau\right) \\
\frac{\partial}{\partial t}\left(\Gamma^{2} \tau-a^{2} \delta^{2} \tau\right)+L_{2} \tau-c_{2}^{\prime} \nabla^{2} \psi \\
\quad=-J\left(\psi, \Gamma^{2} \tau-a^{2} \hat{o}^{2} \tau\right)-J\left(\tau, \Gamma^{2} \psi\right)-\frac{f_{0} I I}{\sigma !}
\end{array}\right\},
$$


where the linear operators $L_{1}$ and $L_{2}$ are clefined as

$$
\left.\begin{array}{l}
L_{1} \equiv \frac{\partial}{\partial \lambda}-A^{\prime} \nabla^{4}-c_{3}^{\prime} \nabla^{2} \\
L_{2} \equiv 2 \frac{\partial}{\partial \lambda}-A^{\prime} \nabla^{4}-c_{4}^{\prime} \nabla^{2}
\end{array}\right\}
$$

and $\delta^{2}=f_{0}^{2} /\left(b c_{p} \sigma\right)$. The primed coefficients refer to the nondimensional form of the dimensionally defined quantities whose values are listed in the Appendix. We have also made use of the nondimensional form of $f=2 \mu$ to remove it from the Jacobians and include it with the linear terms.

\section{Spectral representation}

We have chosen to solve system (10) in the spectral domain principally because of the ease with which truncation may be altered therein. Spectral components may be added or subtracted from the system with lit le modification both to the formal relations and to the computational programs. Moreover, the spectral equations have been shown to eliminate aliasing and to conserve integral constraints in truncated, nonforced systems (see, for example, Baer, 1964; Robert, 1966; or Merilees, 1968).

The detailed properties of the polynomials used in the spectral expansion of variables on a spherical surface (spherical harmonics) and their applicability to meteorological problems have been discussed by Platzman (1960) ; consequently, any aspect of the following discussion which may appear too cryptic to the reader may be clarified by reference to that paper.

We define the mean and shear streamfunctions in series of Legendre polynomials as

$$
\left.\begin{array}{c}
\psi=\sum_{\gamma} \psi_{\gamma}(l) Y_{\gamma}(\lambda, \mu) \\
\tau=\sum_{\gamma} \tau_{\gamma}(l) Y_{\gamma}(\lambda, \mu)
\end{array}\right\},
$$

where $\gamma=n_{\gamma}+i l_{\gamma}$ represents a wave vector index in terms of rank and degree of the solid harmonic

$$
Y_{\gamma}(\lambda, \mu)=\exp \left(i l_{\gamma} \lambda\right) P_{\gamma}(\mu)
$$

satisfying the differential equation

$$
\nabla^{2} Y_{\gamma}=-c_{\gamma} Y_{\gamma}, \quad c_{\gamma} \equiv n_{\gamma}\left(n_{\gamma}+1\right),
$$

and with the orthogonal property that

$$
\frac{1}{4 \pi} \int I_{\alpha} Y_{\beta}^{*} d s=\delta_{\alpha, \beta}
$$

Since the functions $\psi$ and $\tau$ are real, the series (12) must extend over both positive and negative values of $l_{\gamma}$; however, it may be shown that conjugate values of the coefficients (denoted by an asterisk) are related such that $\psi_{\gamma}{ }^{*}=\psi_{\gamma}{ }^{*}$ and $\tau_{\gamma}{ }^{*}=\tau_{\gamma}{ }^{*}$.
The conventional procechure to reduce ligs. (10) to spectral form is to multiply each equation by a conjugate polynomial $\left(Y_{\gamma}{ }^{*}\right)$ and integrate over the unit sphere. Because of the orthogonality condition (14), all linear terms will yield a single expansion coefficient, say $\psi_{\gamma}$. The linear operators $I_{1}$ and $L_{2}$ defined in (11) thus yield

$$
\left.\begin{array}{l}
\frac{1}{4 \pi} \int L_{1} \psi Y_{\gamma}{ }^{*} d s=\left(2 i l_{\gamma}-A^{\prime} c_{\gamma}{ }^{2}+c_{3}{ }^{\prime} c_{\gamma}\right) \psi_{\gamma} \equiv c_{\gamma}{ }^{\prime}{ }_{\gamma} \psi_{\gamma} \\
\frac{1}{4 \pi} \int L_{2} \tau Y_{\gamma}{ }^{*} d s=\left(2 i l_{\gamma}-A^{\prime} c_{\gamma}{ }^{2}+c_{1}^{\prime} c_{\gamma}\right) \tau_{\gamma} \equiv \hat{c}_{\gamma} \eta_{\gamma} \tau_{\gamma}
\end{array}\right\},
$$

where we shall define $\hat{c}_{\gamma} \equiv c_{\gamma}+a^{2} \delta^{2}$, a modified eigenvalue discussed in terms of energy exchanges in low-order spectral systems by Baer (1968).

The terms in the Jacobians of (10) are clearly nonlinear and result in interactions between most of the expansion coefficients $\left(\psi_{\gamma}, \tau_{\gamma}\right)$. For ease in representation and programing, we shall follow the procedure recommended by Baer and King (1967). Since we shall always truncate the series for $\tau$ and $\psi(12)$ at the same wave vector $\gamma$, let us consider $\psi_{\gamma}$ and $\tau_{\gamma}$ as a vector pair and represent them by the symbol $\zeta_{\gamma}$, where

$$
\zeta_{\gamma}=\left(\begin{array}{c}
\psi_{\gamma} \\
\tau_{\gamma}
\end{array}\right)
$$

The entire field of $\zeta_{\gamma}$ may then be written as a vector $\zeta$ such that

$$
\zeta=\left(\begin{array}{c}
\zeta_{\gamma_{1}} \\
\zeta_{\gamma_{2}} \\
\vdots \\
\zeta_{\gamma_{\max }}
\end{array}\right)
$$

Because of the nonlinearity of the Jacobians, one must evaluate integrals of three solid harmonics, termed interaction coefficients, which have the following form [for details on these coefficients the reader is referred to Batr and Platzman (1961)]:

$$
K_{\gamma, \alpha, \beta}=\frac{i}{2} \int_{-1}^{1}\left(l_{\alpha} P_{\alpha} P_{\beta}^{\prime}-l_{\beta} P_{\beta} P_{\alpha}^{\prime}\right) P_{\gamma} l_{\mu}
$$

where the prime denotes differentiation with regard to $\mu$ and $i=\sqrt{-1}$. We now develop the following $(2 \times 2)$ matrices incorporating the interaction coefficients defined in (18) and having all zero elements except for that. matrix location defined by the superscript indices; for example,

$$
\mathbf{K}_{\gamma \alpha \beta^{21}} \equiv\left(\begin{array}{cc}
0 & 0 \\
K_{\gamma \alpha \beta} & 0
\end{array}\right) \text {, etc. }
$$

These matrices may be used to develop larger square matrices with the same dimensions as the vector $\zeta$, and ordered on $\alpha$ and $\beta$ in the same sense as (17). Thus, 
we would have

$$
\mathbf{K}_{\gamma}^{i j}=\left(\mathbf{K}_{\gamma \alpha \beta^{i j}}\right) \text {, etc., }
$$

where $1 \leqslant i \leqslant 2$ and $1 \leqslant j \leqslant 2$. We must finally define matrices involving the cigenvalues $c_{\gamma}$, since they occur through the Iaplacian in all the Jacobians of (10). Following a procedure similar to (19) and (20), we let the $(2 \times 2)$ diagonal matrix $\overline{\mathbf{C}}_{\gamma}$ be defined as $c_{\gamma}$ times the unit matrix $\mathbf{E}$, i.e.,

$$
\overline{\mathbf{C}}_{\gamma} \equiv c_{\gamma} \mathbf{E}=c_{\gamma}\left(\begin{array}{ll}
1 & 0 \\
0 & 1
\end{array}\right)
$$

Now genclating a spuare matrix with the dimensions of $\zeta$ with $\overline{\mathrm{C}}_{\gamma}$ on the diagonal and ordered as in (17), i.e.,

$$
\mathbf{C} \equiv\left(\begin{array}{cccccc}
\overline{\mathbf{C}}_{\gamma_{1}} & 0 & \cdot & \cdot & \cdot & 0 \\
0 & \overline{\mathbf{C}}_{\gamma_{2}} & \cdot & \cdot & \cdot & 0 \\
\cdot & \cdot & \cdot & \cdot & \cdot & \cdot \\
\cdot & \cdot & \cdot & \cdot & \cdot & \cdot \\
0 & 0 & \cdot & \cdot & \cdot & \overline{\mathbf{C}}_{\gamma_{\text {tasx }}}
\end{array}\right),
$$

we may write all Jacobians in matrix form. Note that the matrix $\hat{C}$ is generated simply by substituting $\hat{c}_{\gamma}$ for $c_{\gamma}$ in (22). As an example,

$$
\frac{1}{4 \pi} \int Y_{\gamma}{ }^{*}\left[J\left(\tau, \nabla^{2} \psi\right)\right] d s=\tilde{\zeta} \mathbf{C K}_{\gamma}{ }^{12} \zeta,
$$

where the tilde denotes transposition. Finally, we define the contribution to components from the heating function (which we shall discuss in detail subsequently) as

$$
-\frac{1}{\hat{c}_{\gamma} 4 \pi} \int\left(\begin{array}{c}
f_{0} H \\
\hdashline \Omega \\
\sigma \Omega
\end{array}\right) Y_{\gamma} * d s=M_{\gamma} .
$$

Utilizing the definitions (12) (2t), we may now write Eqs. (10) in spectral form as

$$
\dot{\zeta}_{\gamma}-\mathbf{B}_{\gamma} \zeta_{\gamma}=\left(\begin{array}{l}
1 \\
-\tilde{\zeta} \mathbf{C}\left(\mathbf{K}_{\gamma}{ }^{11}+\mathbf{K}_{\gamma}{ }^{22}\right) \zeta \\
c_{\gamma} \\
1 \\
-\tilde{\zeta}\left(\hat{\mathbf{C}} \mathbf{K}_{\gamma}{ }^{21}+\mathbf{C K}_{\gamma}{ }^{12}\right) \zeta+M_{\gamma}
\end{array}\right)=\mathbf{R}_{\gamma},
$$

where

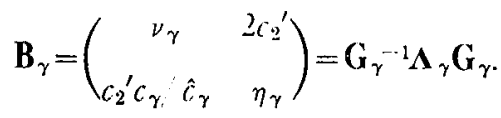

In (26) $\mathbf{G}_{\gamma}$ is the modal matrix of $\mathbf{B}_{\gamma}$, and $\boldsymbol{\Lambda}_{\gamma}$ represents the eigenvalue matrix. Since the elements of $\mathbf{B}_{\gamma}$ are invariant in time, the eigenvalues of each matrix may be evaluated once and for all. Note how clearly the time rate of change of any element $\zeta_{\gamma}$ may be interpreted from (25) in terms of its dependence on the quadratic relationship of the entire $\zeta$ vector, whereas the linear dependence is entirely incorporated in the matrices $\mathbf{B}_{\gamma}$. The elements $M_{\gamma}$ will be seen to be inhomogeneous.
System (25) represents a set of ordinary, nonlincar differential equations in time. For any reasonable truncation of wave vectors $\gamma$, these equations must be solved numerically. Because of its simplicity and success in previous experiments, we have chosen the leapfrog scheme as our integration procedure. Noting the reduction in truncation attained by treating the linear terms exactly (Baer and Simons, 1970), we extrapolate numerically the equation

$$
\exp \left(\mathbf{B}_{\gamma} t\right) \frac{d}{d t}-\left[\exp \left(-\mathbf{B}_{\gamma} t\right) \zeta_{\gamma}\right]=\mathbf{R}_{\gamma}
$$

which leads to the result that

$$
\zeta_{\gamma}{ }^{t+\Delta t}=\exp \left(2 \mathbf{B}_{\gamma} \Delta t\right) \zeta_{\gamma}{ }^{t-\Delta t}+2 \Delta l \exp \left(\mathbf{B}_{\gamma} \Delta l\right)
$$

where we make use of (26) to evaluate the matrix exponential. We note that (28) is a threc-level system in time (Richtmyer, 1957) and is therefore subject to parasitic modes of period $2 \Delta t$. These oscillations are inhibited by using a restart technique (Kikuchi, 1969) whereby the system (27) is periodically (every 96 time steps) treated as a two-level system and integrated one time step with the Euler forward scheme.

Finally, the model is integrated on the hemisphere rather than the entire sphere. This is achieved by requiring that the vectors in the expansion of $\zeta$ are all of odd parity, thereby making the streamfunction an odd function of latitude. From the geostrophic relation it may be seen that this recuires the zonal wind to be symmetric across the equator whereas the meridonal wind component will be antisymmetric.

The reader should note that spectral truncation is achieved simply by defining the vectors (and their order) in the stream field $\zeta[\mathrm{Eq} .(17)]$. Since all matrices depend on the truncation and order of $\zeta$, no modification in the extrapolation equation (28) is required for different 1 runcations. It will be seen, however, that the time increment $(\Delta t)$ must be varied for different spectral truncations.

\section{Forcing and energetics}

'To maintain simplicity we have chosen to drive our model atmosphere with a heating function which is purely inhomogeneous, in a manner similar to Phillips (1956). Although the heating function would be more realistic if it were related to the dependent thermal variables---a point explored with reference to numerical models many years ago by (harney (1959) - the problem of truncation may be investigaterl without this complexity.

Fig. 1 describes a number of estimates of atmospheric diabatic heating, including all heat sources as a function of latitude in langleys per day. These data include values used by different modellers (Phillips, 1956; Mint 2,1958 ; Smagorinsky of al., 1965), and estimates taken from observation (I)avis, 1963; Lettau, 
1954; Sellers, 1965 ; Lorenz, 1967). It is clear from the figure that a wide range of values exists with the common effect that there is heating in the tropies and cooling at the pole. More detailed data are currently being taken by systematic satellite measurements, but broad variations in these data do not lead to a more valid curve for Fig. 1. Consequently, we have elected to use the heating function given by the solid curve on Fig. 1, made up of only two zonal polynomials $(\gamma=2,4)$ and represented as

$$
H(\mu)=h_{2} P_{2}(\mu)+h_{4} P_{4}(\mu),
$$

a function which vanishes when integrated from pole to equator. The values of $h_{2}$ and $h_{4}$ are listed in the $\Lambda$ ppendix in dimensional form to correspond to the values plotted on Firs. 1. ("learly $I I$ as described by (29) is an even function of latiturle. However, we note that $H$ enters linearly into (10), the equation for prediction of the stream shear. If we are to maintain a purely odd system in the mean and shear streamfunctions as indicated earlier (i.e., allow only odd wave vectors $\gamma$ ), $H$ must enter in (10) as an odd function. Such a form may be constructed as follows. The heating function $H$ as it enters (10) is preceded by a coefficient which represents a mean value of the Coriolis parameter and was established by the elimination of the vertical velocity $\left(\omega_{2}\right)$ between the iirst and third equations of (3). A mean ('oriolis parameter was chosen in (3) in order to assure that the non-forced, non-dissipative system was energetically conservative. Since the heating function is clearly not constrained by a conservation condition, we may allow $f$ to vary when it multiplies the heating function. Since $f$ is an odd function and is proportional to $\mu$, we substitute $f H$ for $f_{0} H$ in (10). The desired function now becomes $\mu I I$, an odd function, given as

$$
\left.\begin{array}{rl}
\mu I I & ={ }_{\sqrt{15}-h_{2} P_{1}}+\frac{1}{\sqrt{7}}\left(\frac{3}{\sqrt{5}}+\frac{t}{3}+h_{2}+h_{4}\right) P_{3}+\frac{5}{3 \sqrt{11}} h_{4} P_{3} \\
& =\frac{\sigma}{2} \sum_{j=0}^{2} \hat{c}_{2 j+1} M_{2 j+1}
\end{array}\right\},
$$

by use of (29), (24) and the recursion formulas for Legendre polynomials (see, for example, Hobson, 1955). Eq. (30) is described graphically on Fig. 1 by the dashed curv; but care should be used in its interpretation; it represents the function used in the prediction equation for the shear streamfunction but is not specifically the heating function for our model, which is given by the solid curve.

The energy response of our model to the heating function discussed above will undoubtedly be of great significance in interpreting the truncations used. Since the model is treated in the spectral domain we may represent the energy in wave components immediately by the definitions

$$
\left.\begin{array}{c}
\bar{K}_{\gamma} \equiv c_{\gamma} \psi_{\gamma} \psi_{\gamma}{ }^{*} \\
K_{\gamma}{ }^{\prime} \equiv c_{\gamma} \tau_{\gamma} \tau_{\gamma}{ }^{*} \\
A_{\gamma} \equiv a^{2} \delta^{2} r_{\gamma} \tau_{\gamma}{ }^{*} \\
K_{\gamma} \equiv \bar{K}_{\gamma}+K_{\gamma}^{\prime}
\end{array}\right\},
$$

where $\bar{K}_{\gamma}$ represents the mean kinetic energy, $K_{\gamma}^{\prime}$ represents the shear energy, $K_{\gamma}$ is the total kinetic energy; and $A_{\gamma}$ represents the available potential energy, all in the $\gamma$ component. The total energy in each of these categories is clearly

$$
\left.\begin{array}{l}
\bar{K}=\frac{1}{2} \int \Gamma \psi \cdot \Gamma \psi d s=\frac{1}{2} \sum_{\gamma} \bar{K}_{\gamma} \\
K^{\prime}=\frac{1}{2} \int \Gamma \tau \cdot \Gamma \tau d s=\frac{1}{2} \sum_{\gamma} K_{\gamma}^{\prime} \\
\begin{array}{l}
1 \\
=\frac{1}{2}
\end{array} \int a^{2} \delta^{2} \tau^{2} d s=\int \frac{a^{2} b c_{p}}{2 \sigma} \theta^{2} d s=\frac{1}{2} \sum_{\gamma} A_{\gamma} \\
E=\bar{K}+K^{\prime}+A=\frac{1}{2} \sum_{\gamma}\left(c_{\gamma} \psi_{\gamma} \psi_{\gamma}{ }^{*}+\hat{c}_{\gamma} \gamma_{\gamma^{\prime}} \gamma^{*}\right)
\end{array}\right\}
$$

It has already been made evident that the total energy $E$ of our model must be conserved except for heating and dissipation. There are also exchanges occurring between different forms of energy in this model, and they may be seen from the differential equations (3). Using these equations, we get for the time rate of change of energy:

$$
\begin{aligned}
& \frac{\partial \bar{K}}{\partial t}=-\frac{1}{4 \pi} \int \psi \frac{\partial \nabla^{\prime \prime} \psi}{\partial t}--d s=\frac{1}{4 \pi} \int \psi L_{1} \psi d s \\
& -\frac{2 c_{2}^{\prime}}{4 \pi} \int \psi \Gamma^{\prime \prime} \tau d t s+\frac{1}{4 \pi} \int \psi J\left(\tau, \Gamma^{2} \tau\right) d s \\
& \frac{\partial K^{\prime}}{\partial l}=-\frac{1}{4 \pi} \int \tau-\frac{\partial \nabla^{2} \tau}{\partial t} d s=\frac{1}{4 \pi} \int \tau L_{2} \tau d l s \\
& -\frac{c_{2}^{\prime}}{4 \pi} \int \tau \Gamma^{\prime \prime} \psi d s+\frac{1}{4 \pi} \int \tau J\left(\psi, \Gamma^{\prime \prime} \tau\right) d s \\
& \left.\frac{2 f_{0}}{4 \pi p_{0}, \Omega^{2}} \int \tau \omega_{\mathrm{g}} / s\right\}
\end{aligned}
$$

$$
\begin{aligned}
& \frac{\partial A}{\partial t}=\frac{1}{4 \pi} \int a^{2} \hat{\delta}^{2} \tau \frac{\partial \tau}{\partial t} d s=\frac{1}{4 \pi p_{0} \Omega^{2}} \int \tau \omega_{0} d s \\
& +\frac{1}{+\pi \sigma \Omega} \int j H \tau d s \\
& \frac{\partial E}{\partial t}=\frac{1}{4 \pi} \int\left(\psi L_{1} \psi+\tau L_{2} \tau\right) d s \\
& -\frac{3 c^{\prime}}{4 \pi} \int \psi \nabla^{2} \tau d s+\frac{2}{4 \pi \sigma \Omega} \int f H \tau d s
\end{aligned}
$$


From (33) we see that exchange of kinetic energy between the mean and shear occurs through the Jacobian terms, whereas conversion of potential to kinetic energy: takes place through the correlation of $\omega_{2} \theta$. Moreover, this conversion can contribute directly only to the shear kinetic energy. The last of Eys. (3.3) shows, as already indicated, that variations in total energ. come about only by heating and clissipation (diffusion or momentum exchange with the ground). The terms entering the equation for the time change of $E$ may be evaluated in spectral form by use of (12) and (15) to yield

$$
\begin{gathered}
\frac{1}{4 \pi} \int\left(\psi L_{1} \psi+\tau L_{2} \tau\right) d s=\sum_{\gamma}\left[\left(-c_{\gamma} \cdot 1^{\prime}+c_{3}^{\prime}\right) \bar{K}_{\gamma}\right. \\
\left.+\left(-c_{\gamma} 1^{\prime}+c_{4}^{\prime}\right) K_{\gamma}^{\prime}\right] \\
\frac{3 c_{2}^{\prime}}{4 \pi} \int \psi \nabla^{2} \tau d s=-3 c_{2}^{\prime} \sum_{\gamma} c_{\gamma} \psi_{\gamma} \tau_{\gamma}{ }^{*} \\
-\frac{1}{4 \pi \sigma \Omega} \int f I I \tau d s=\sum_{j=11}^{2} \hat{c}_{2 j+1} M_{2 j+1} \tau_{2 j+1}
\end{gathered}
$$

Noting that $c_{1}{ }^{\prime}, c_{2}^{\prime}$ and $d^{\prime}$ are all positive quantities, and utilizing the clefinitions of $c_{3}{ }^{\prime}$ and $c_{1}{ }^{\prime}$ from Section 2 , we find for the coeflicients in the first of $(34)$

$$
\left.\begin{array}{l}
c_{3}^{\prime}-c_{\gamma} A^{\prime}=-\left[c_{2}^{\prime}+A^{\prime}\left(c_{\gamma}-2\right)\right]<0 \\
c_{1}^{\prime}-c_{\gamma} A^{\prime}=-\left[2 c_{1}^{\prime}+c_{2}^{\prime}+\left(c_{\gamma}-2\right) \cdot 1^{\prime}\right]<0
\end{array}\right\},
$$

since by choosing only odd vectors $\gamma, c_{\gamma} \geqslant 2$.

Eqs. (3t) and (35) lead to the following observations about the energetics of our model. The internal stress, as represented by $c_{1}{ }^{\prime}$, always acts to reduce the shear kinetic energy whereas the horizontal diffusion $A$ 'acts 10 reduce both the mean and shear kinetic energ!: (learly the heating as represented by the three coefficients $M_{\gamma}$ tends to increase the available potential energy, but only in the three lowest modes of the zonal field. Finally, although surface stress generally acts to reduce the kinetic energy of both the mean and shear, there is a special condition under which momentum may actually be added to the atmosphere by this process. This comes about if the vorticity at level three exceeds the vorticity at level one by more than a factor of 3 , i.e., when $\zeta_{3}>3 \zeta_{1}$. In spectral terms this comes about when

$$
\sum_{\gamma} c_{\gamma}\left(2 \tau_{\gamma}-\psi_{\gamma}\right)\left(\psi_{\gamma}-\tau_{\gamma}\right)^{*}>0
$$

a condition which follows readily from (34) when applied to (3.3). Indeed this effect is a conserpuence of choosing the surface wind by linear extrapolation from levels 3 and 1 .

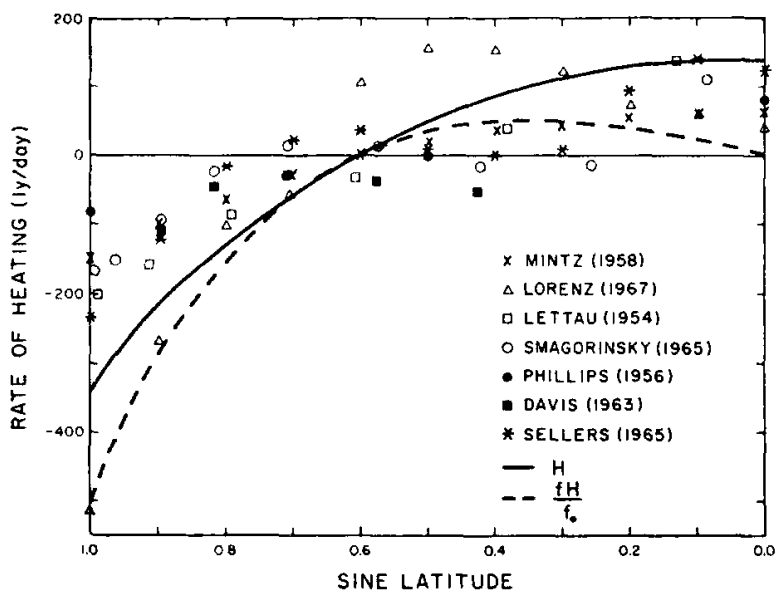

lig. 1. Various heating rates (ly day-1) used in general circulation molels and laken from olsservations, including the one used in the present model, as a function of latitude.

\section{Truncation and initialization}

Truncation in the spectral domain is defined by the approximation of the infinite series (12) to a finite series which includes $I$ wave vectors $\gamma$. We then note from (17) that there will be $2 N$ unknowns (degrees of freedom) in the system with $\gamma_{\text {max }}=\gamma_{N}$. By choosing different values of $Y$ and solving (28), we may investigate the effect of spectral truncation. There are, however, many ways to limit $\gamma$ as we have already seen it to depend on two quantities $l_{\gamma}$ and $n_{\gamma}\left(\gamma=n_{\gamma}+i l_{\gamma}\right)$. From their relation to the Legendre polynomials, $l_{\gamma}$ represents the planetary wavenumber and $n$ the degree of the polynomial. Furthermore $n_{\gamma}$ must be greater than $\left|l_{\gamma}\right|,\left(n_{\gamma}>\left|l_{\gamma}\right|\right)$, and both must be integers. Finally, since we allow for only odd functions, $n_{\gamma}+l_{\gamma}$ must be odd. With these limitations on $n_{\gamma}$ and $l_{\gamma}$, we may describe the allowed vectors $\gamma$ for a given truncation on an $n-l$ diagram with $l$ on the abscissa and $n$ on the ordinate, somewhat analogous to a rotated complex plane. All allowed vectors in a truncation set (hereinafter to be referred to as an experiment) emanate from the origin and terminate at the intersection of the integers $(n, l)$. 'Two such vectors, $\gamma=14-11 i$ and $\gamma=19+4 i$, are described as examples on Fig. 2.

In previous studies utilizing the spectral method several truncation schemes were applied, of which the two most popular were the triangular (Baer, 196-t) and the rhomboidal (Elsaesser, 1966). Based on the successful integrations of Elsaesser, we have chosen to use rhomboidal truncation. The conditions for a rhomboidal experiment require the specification of a maximum planetary wavenumber $\left(l_{\text {nux }}\right)$ and an integer $(m)$ to denote the number of vectors for a given $l_{r}$ in the $n$ direction. We thus have for each experiment all $\gamma$ vectors included in the set

$$
\left.\begin{array}{l}
0 \leqslant l_{\gamma} \leqslant l_{\max } \\
n_{\gamma}=l_{\gamma}-1+2 j, \quad \text { for } \quad 1 \leqslant j \leqslant m+\hat{j}_{l, .0}
\end{array}\right\} .
$$




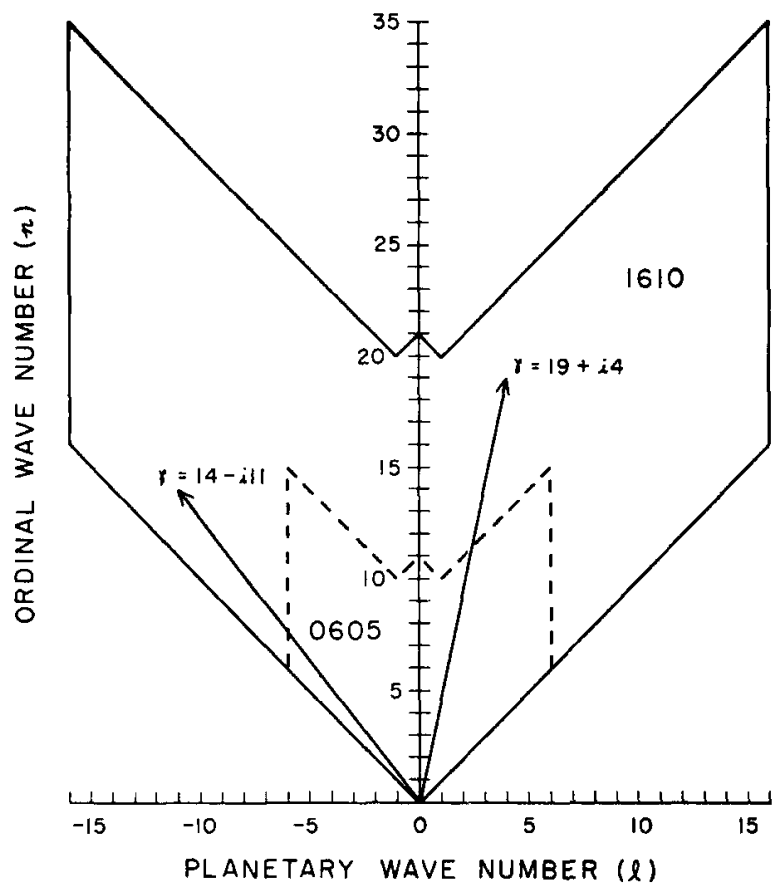

lici. 2. Spectral map inclurling the wave vector bounclaries of two truncation experiments and two sample wave vectors in $n, l$ coordinates.

Conditions (36) imply that the domain of $\gamma$ vectors of any experiment as represented on the $n-l$ diagram be completely filled, i.c., without holes. Examples of two such experiments for the smallest and largest truncations utilized in our study are depicted on Fig. 2 where the limiting values $n_{\max }(l)=l-1+2 m+2 \delta_{l, 0}$ from $l=0$ to $l_{\max }$ are connected and enclose the region of included wave vectors $\gamma$. The extra vector in the zonal field $(l=0)$ is based on the fact that the stream coefficient for $\gamma=1$ is inactive in a nonforced barotropic system.

Given the limits $\left(l_{\text {uax }}, m\right)$ for a given experiment $(I D)$, we may now order the vectors $\gamma$ as follows. Beginning with $l_{\gamma}=0$, the vectors are ordered on increasing $n_{\gamma}$ to $n_{\gamma}(\max )$ for $l_{\gamma}=0$ and then increasing $l_{\gamma}$ to $l_{\max }$. More specifically we have

$$
\begin{gathered}
\gamma_{0}=1, \quad \gamma_{1}=3, \ldots, \quad \gamma_{m}=2 m+1 \\
\gamma_{m+1}=2+i, \ldots, \quad \gamma_{2 m}=2 m+i \\
\gamma_{2 m+1}=3+2 i, \ldots, \quad \gamma_{l_{m+j}}=l-1+2 j+l i, \ldots, \\
\gamma_{\left(l_{\max }+1\right) m}=l_{\max }-1+2 m+l_{\max } i .
\end{gathered}
$$

This ordering uniquely specifies the position of any variable $\zeta_{\gamma}$ in the vector $\zeta[\mathrm{Eq} .(17)]$. The vectors with negative planetary wavenumber $\left(l_{\gamma}<0\right)$ are not included in the set because of the previously stated condition that $\psi_{\gamma}{ }^{*}=\psi_{\gamma}{ }^{*}$, which identifies all required information in the domain of positive wavenumbers. These vectors exist, however, and the total number of such vectors in a given experiment is $2 m\left(l_{\text {max }}+\frac{1}{2}\right)+1$, whereas the number in the above set is $m\left(l_{\max }+1\right)+1$.
Nine experiments (spectral truncations) were selected for study ranging from 132 to 662 degrees of freedom. They are listed, together with selected properties, in Table 1. Among these properties we inclucle $l_{\max }, m$, $n_{\text {max }}$, total number of wave vectors $\gamma$ (one-half the number of degrees of freerlom), the total integration period in days, the time increment $(\Delta t)$ in hours used for extrapolation (and found to be computationally stable), and finally the number of unique, nonzero interaction coefficients, $K_{\gamma, \alpha, \beta}[\mathrm{E}(1$. (18) $]$. Since the latter constants are used multiplicatively at each time step in the matrix multiplications indicated in (28) by $\mathbf{R}_{\gamma}{ }^{2}$, one sees the dramatic increase in computation with the increase in spectral resolution. Adding to this increase we furthermore note that the addition of more wave vectors (including shorter, faster traveling waves) requires a smaller extrapolation interval $(\Delta t)$, thereby compounding the computational requirements.

Since we are dealing here with a nonlinear initial value problem, each experiment requires the specilication of an initial state before the extrapolation may be performed. Moreover, as we are interested in spectral truncation, all experiments should begin with the same initial state. This state may be chosen from a variety. of states which are characteristic of the atmosphere. Such a choice, however, would leave the ultimate conclusions dependent on that choice of initial conditions. Fortunately, as with all general circulation experiments, we are dealing with a forced initial value problem which we may assume will generate its own initial conditions starting from a state of rest, a procedure followed by most general circulation modellers. Such an initial state is specified for our spectral model simply by requiring each element of the vector $\zeta(t=0)=0$ [see Eq. (17)], implying no motion at the initial time and a temperature specified by the choice of static stability; note that Eq. (2) relates $\theta$ and $\tau$ only to within an arbitrary constant.

Unfortunately, it can be shown that if there is no amplitude in the wave components $\gamma$ for $\gamma$ complex, all nonlinearity vanishes and we are left with a forced, uncoupled linear system. This comes about because

\begin{tabular}{|c|c|c|c|c|c|c|c|}
\hline$I D$ & $l_{(\max )}$ & $n_{(\mathbf{m} ; \mathbf{x})}$ & $m$ & $\begin{array}{c}\text { Number } \\
\text { of } \\
\text { vectors }\end{array}$ & $\begin{array}{l}\text { Period } \\
\text { (rlays) }\end{array}$ & $\begin{array}{c}\Delta l \\
(\mathrm{hr})\end{array}$ & $\begin{array}{l}\text { Number of } \\
\text { interaction } \\
\text { coefficients }\end{array}$ \\
\hline 0605 & 6 & 15 & 5 & 66 & 180 & 1.5 & 3118 \\
\hline 0805 & 8 & 17 & 5 & 86 & 156 & 1.5 & 5416 \\
\hline 0807 & 8 & 21 & 7 & 120 & 156 & 1.5 & 14000 \\
\hline 1006 & 10 & 21 & 6 & 127 & 158 & 1.5 & 14032 \\
\hline 1208 & 12 & 27 & 8 & 201 & 96 & 0.75 & 45788 \\
\hline 1408 & 14 & 29 & 8 & 2.33 & 96 & 0.75 & 0233.9 \\
\hline 1608 & 10 & 31 & 8 & 26.5 & 96 & 0.75 & 814.50 \\
\hline 1410 & 14 & 33 & 10 & 291 & 96 & 0.50 & 118241 \\
\hline 1610 & 16 & 35 & 10 & 331 & 96 & 0.40 & 154946 \\
\hline
\end{tabular}
of the conditions for nonvanishing of the interaction

Table 1. Pertinent data describing the truncation properties of the various experiments for which integralions were performed. 
coefficients $K_{\gamma, \alpha, \beta}$ (see Baer and Platzman, 1961). One of these conditions requires that $l_{\gamma}=l_{\alpha}+l_{\beta}$, which requires either all $l$ 's to vanish or that at least two be non-zero. The former condition leads to no interaction since we see from (18) that these interaction coefficients vanish. The latter condition does indeed yield nonvanishing interaction coefficients, but at least one of the elements in each matrix product $\mathbf{R}_{\gamma}[\mathrm{Eq} .(25)]$ vanishes by nature of the fact that $\zeta_{\gamma}=0$ for all $\gamma$.

Let us now consider the linear equations which result from the assumption that $\zeta(t=0)=0$. From (25) wc have

$$
\dot{\zeta}_{\gamma}-\mathbf{B}_{\gamma} \zeta_{\gamma}=\mathbf{R}_{\gamma} \equiv\left(\begin{array}{c}
0 \\
M_{\gamma}
\end{array}\right)
$$

thus yielding a separate solution for each couple $\zeta_{\gamma}$. I) iagonalizing $\mathbf{B}_{\gamma}$ by a similarity transformation, we have the solution for $\zeta_{\gamma}$ :

$$
\left.\begin{array}{l}
\zeta_{\boldsymbol{\gamma}}=\mathbf{G}_{\boldsymbol{\gamma}}{ }^{-1}\left[\exp \left(\mathbf{\Lambda}_{\gamma} l\right)-\mathbf{E}\right] \mathbf{\Lambda}_{\boldsymbol{\gamma}} \mathbf{G}_{\boldsymbol{\gamma}} \mathbf{R}_{\gamma} \\
\mathbf{B}_{\boldsymbol{\gamma}}=\mathbf{G}_{\boldsymbol{\gamma}}{ }^{-1} \mathbf{\Lambda}_{\boldsymbol{\gamma}} \mathbf{G}_{\boldsymbol{\gamma}}
\end{array}\right\},
$$

where $\boldsymbol{A}_{\gamma}$ is a diagonal matrix of eigenvalues, $\mathbf{G}_{\gamma}$ the modal matrix, and $\mathbf{E}$ the unit matrix. Clearly we shall have non-zero solutions only for couples $\zeta_{\gamma}$ for which the forcing function coefficients $M_{\gamma}$ are non-zero. Reference to (30) shows that for the heating function selected here, only $\zeta_{\gamma}$ components for $\gamma=1,3,5$ will be activated to set up a forced zonal distribution. The limiting solutions are

$$
\zeta_{\gamma}(t \rightarrow \infty)=-\mathbf{B}_{\gamma}^{-1} \mathbf{R}_{\gamma} .
$$

Computation of (39) for values given in the Appendix show that the mean kinetic energy, when represented as a characteristic velocity, is in the neighborhood of $250 \mathrm{~m} \mathrm{sec}-1$, an unreasonably large value for our atmosphere. This asymptotic solution also shows that $\psi_{\gamma} / \tau_{\gamma} \rightarrow 2$, a condition imposed by the surface stress and the linear extrapolation of the surface stream field from levels 1 and 3. This limiting condition, moreover, takes many days to be realized; the roots $\boldsymbol{\Lambda}_{\gamma}$ (all of which are negative) give $e$-folding times ranging from 4 to 740 days.

Characteristic atmospheric energy values expressed as velocities of $20-30 \mathrm{~m} \mathrm{sec}^{-1}$ are realized from (38) between 30-40 days. We may therefore anticipate that wave activity should begin in this time range to offset the unrealistic growth in the zonal flow. To check the validity of this hypothesis, we have integrated the 0605 experiment with all mean components $\psi_{\gamma}$ given the values of $10^{-5}$ in nondimensional units and the shear $\tau_{\gamma}$ set to zero, i.e., $\psi_{\gamma}=10^{-5}(1+i), \tau_{\gamma}=0$ for all complex $\gamma$. Results of the integration show that the numerical solution follows the solution of (38) exactly for the first 36 days, after which the waves become active and the nonlinear solution diverges significantly from the linear one. The zonal velocity distribution of both mean and shear at day 36 is shown on Fig. 3.

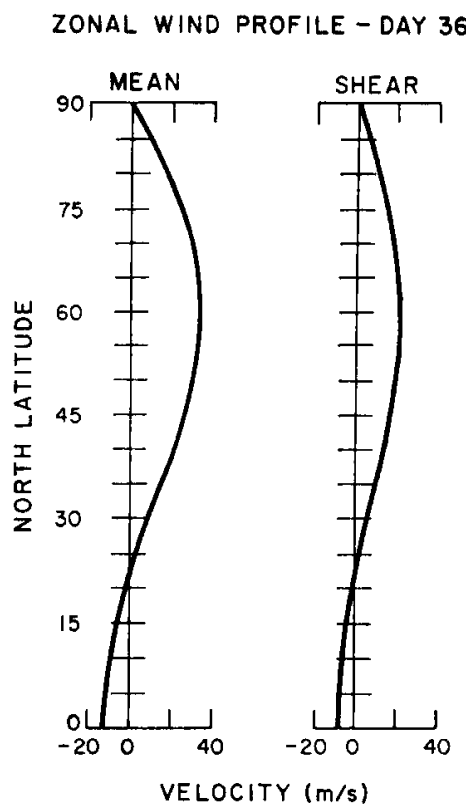

FJg. 3. Zonal wind profile for mean and shear $\left(\mathrm{m} \mathrm{sec}^{-1}\right)$ generated by zonal forcing at day 36 and used as initial conditions.

Since the waves are not activated before day 36 , we have chosen to use the distribution of the 0605 experiment at this time (day 36) as the initial conditions for all the truncations listed in Table 1. 'This procedure not only systematizes the initiation of all experiments but does so based on the forced solution required by the heating function. In the subsequent analyses we have assumed for convenience that all experiments begin at $t=0$, but the reader should be aware that actual numerical calculations begin at day 36 with the initial conditions tabulated in the Appendix and specified above. It should also be apparent from the discussion of computing time (earlier in this section) that this initializing procedure afforded considerable savings.

\section{Analysis of data}

In presenting the results of our nine truncation experiments, we shall focus primary attention on the behavior of the energy with time, both the total kinetic energy $K$ and the available potential energy $A$ [see Eqs. (31) and (32)], although the zonal velocity distribution with latitude will also be displayed. We have already seen from (31) that the energy is available in wave component form, i.e, as a function of the wave vector $\gamma$. It is customary as well as meaningful to separate the energy into both a zonal and an eddy part. Such a grouping in terms of our spectral notation implies that

$$
\left.\begin{array}{l}
\text { Zonal } \mathrm{KE} \equiv Z K E=\sum_{\gamma} K_{\gamma}\left(l_{\gamma}=0\right) \\
\text { Eddy } \mathrm{KE} \equiv E K E=K-Z K E=\sum_{\gamma} K_{\gamma}\left(l_{\gamma} \neq 0\right)
\end{array}\right\},
$$

with a similar interpretation for zonal available po- 
TABLE 2. Sixty-day average percent kinetic energy in basic subset (0605) to total kinctic energy, standard deviations, and percent degrees of freedom included in the subset for each experiment.

\begin{tabular}{|c|c|c|c|c|c|c|c|c|c|}
\hline & 0505 & 0805 & 0807 & 1006 & $\begin{array}{l}I D \\
1208\end{array}$ & 1408 & 1608 & 1410 & 1610 \\
\hline $\begin{array}{l}\text { I'ercent degrees of } \\
\text { freedom in subset }\end{array}$ & 100 & 77 & 55 & 52 & 33 & 28 & 25 & 23 & 20 \\
\hline $\begin{array}{l}\text { Percent energy in subset } \\
\text { (time mean) }\end{array}$ & 100 & 89.2 & 87.9 & 86.4 & 90.2 & 91.6 & 91.7 & 94.8 & 93.9 \\
\hline Standaril deviation & $\ldots$ & 6.0 & 5.9 & 6.2 & 4.9 & 4.1 & 3.9 & 2.6 & 1.6 \\
\hline
\end{tabular}

tential energy $(Z A P L)$ and eddy available potential energy (KAPL). Clearly the elements comprising the zonal energy are independent of longitude [equation following (12) ], whereas the eddy elements describe the composite of planetary waves. The energy in each of the latter waves may als') be discussed separately without reference to its latitudinal dependence by the definitions

$$
\left.\begin{array}{l}
K_{l} \equiv \sum_{n \gamma} K_{\gamma}(l) \\
A_{l} \equiv \sum_{n_{\gamma}} A_{\gamma}(l)
\end{array}\right\},
$$

where $K_{l}$ is generally referred to as the energy in planelary wavenumber $l$ and a similar interpretation may be given .4 . Such usage has substantial precedent in meteorological literature and is the basis for many analyses of atmospheric data as exemplified by the works of Saltzman (1958), Wiin-Nielsen (1967) and many others. To describe the energy in each spectral component $\gamma$, we have plotted energy values at the appropriate vector location point on charts such as Fig. 2 (also sometimes termed $n-l$ diagrams), analyzed the clata and generated "spectral maps." Such maps show at a glance the distribution of energy as a function of spectral component and may be prepared from data at any instant of a calculation or for time-averaged data.

Almost total emphasis in analyzing our results will be on comparing the nine different experiments to determine whether increased spectral resolution leads to similarity of solutions. Nevertheless, the tendency to evaluate solutions in terms of their fidelity to observation is overwhelming and we shall therefore include some general comments here and neglect them in subsequent evaluations, which will be directed to the above stated objective. Because of the model's simplicity, the latitude of the principal jet is considerably too far north as seen from Fig. 6. The zonal kinetic energy tends to be overly large by comparison to the wave kinetic energy, especially for the experiments with higher resolution. Within a given planetary wave the energy distribution shows a maximum for $n_{\gamma}$ values midway between the permissible extremes. This feature has yet to be discussed for atmospheric data. The available potential energy for most experiments tends to be about twice the kinetic energy, a result somewhat more in line with observation. Despite these shortcomings of our model, the influences of truncation, as they become apparent, should be indicative of sinilar effects in more sophisticated models.

Current studies of predictability indicate a finite time limit for accurate detailed forecasts, a limit which may not depend directly on truncation (see, for example, Lorenz, 1969). However, the average properties (general circulation) of a forced model may be strongly influenced by truncation. We have therefore separaled our analysis into two parts: one in which we compare results for 30 day averages of the integration data, and the other wherein we compare the detailed structure of variables with time for the first 25 days (beginning, of course, at day 36 as implied by our discussion of initialization).

We shall see subsequently that increasing resolution has a pronounced effect on the ultimate solution, at least until a minimum resolution is reached. Because additional resolution implies more degrees of freedom-more wave components and because all wave components exchange energy nonlinearly [Eq. (25)], one might anticipate that for higher resolution experiments energy would spread over the entire spectral domain and less energy would be found in the subset of components comprising the lowest truncation $(I D=0605)$, thereby providing at least some explanation for differences in solution. To test this hypothesis, Table 2 was prepared, wherein we describe for each experiment the time average percent of kinetic energy in a basic subset (henceforth to be considered the set of components included in the 0605 experiment) to the total kinetic energy together with its standard deviation, and the ratio of degrees of freedom in the subset to the total number (this latter information is taken from Table 1). The time period for which averages are taken includes day 36 to day 95 . Remarkably, we find from this table that more of the energy is confined to the basic subset for the experiments with largest resolution despite the small percentage of components involved. We are thus led to the initial conclusion that the addition of degrees of freedom to a system does not act as an energy drain to the basic subset but has a catalytic effect which allows for a redistribution of energy among the components in the basic subset. Moreover, since such a high percentage of energy remains in the basic subset, we may make comparisons of the different experiments by references to this subset alone. 

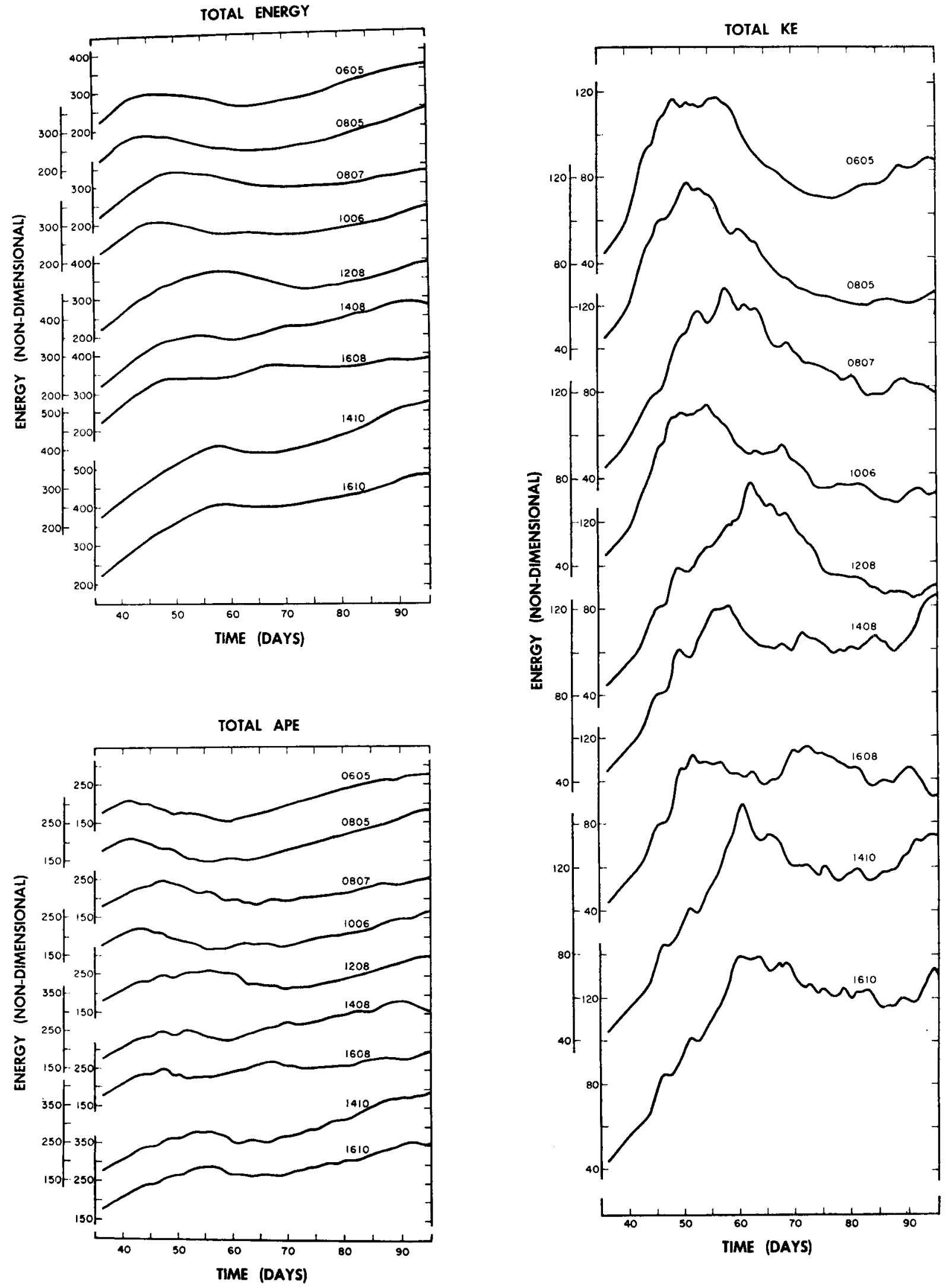

Fio. 4. Total energy, total available potential energy and total kinetic energy in nondimensional units as a function of time to day 95 for all nine truncation experiments. 

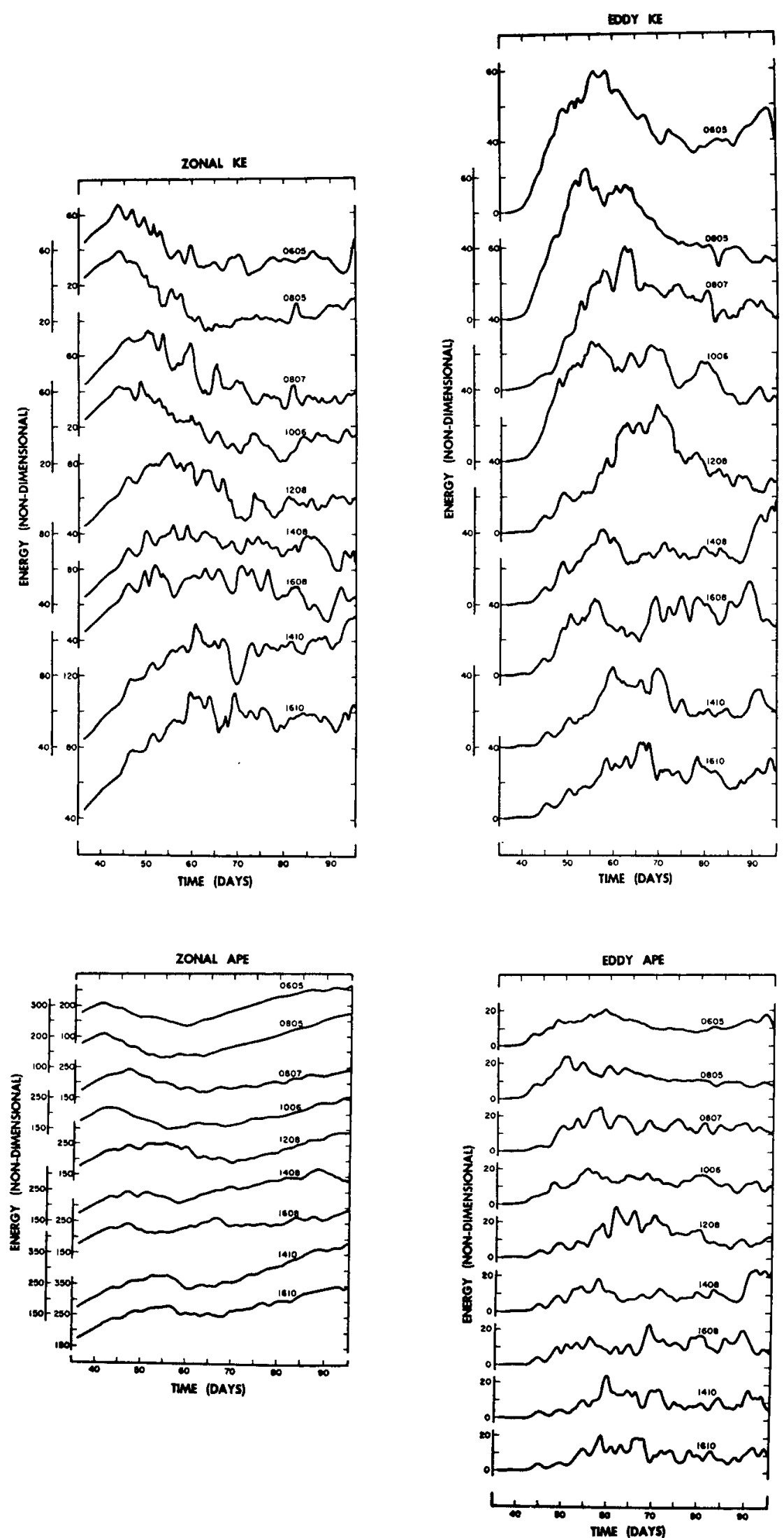

Fig. 5. Zonal and eddy kinetic and availahle potential energy in nondimensional units as a function of time to day 95 for all nine truncation experiments. 

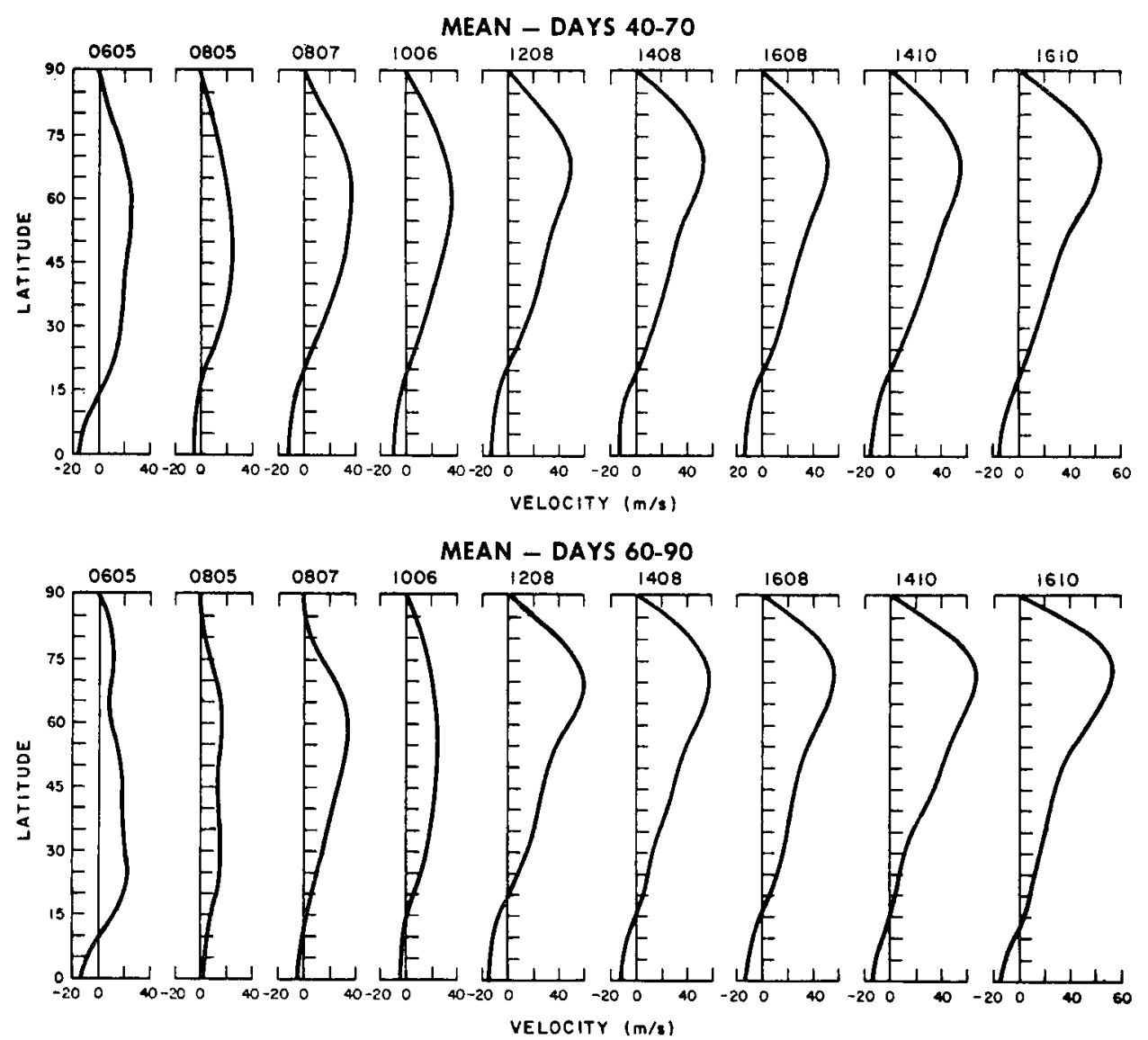

Fig. 6. Thirty-clay mean zonal velocity profiles $\left(\mathrm{m} \mathrm{sec}^{-1}\right)$ as a function of latitude for all nine truncation experiments.

The general structure of the energy field as it develops with time has been displayed in Fig. 4, wherein the total energy $E$, the available potential energy $A$ and the total kinetic energy $K$ are plotted for each experiment from day 36 to day 95 . The curves from top to bottom represent the experiments in order of increasing resolution as they are described in Table 1 . This figure shows that $E$ reaches a relative maximum during the period and then continues to increase for each experiment, although the relative maximum varies from one experiment to another, whereas the level of energy attained increases with increasing resolution. We note here the similarity between the two experiments of maximum resolution (1410 and 1610) but defer interpretation of this observation. The total potential energy develops in time for all experiments in a manner very similar to the total energy despite the fact that it represents only about two-thirds of that energy.

The total kinetic energy, on the other hand, shows marked fluctuations and a decidedly more pronounced maximum, which is reached more than once only by cases 1408 and 1608 , and varies in time from one case to another. Indeed, similarity in time fluctuations are clearly evident only between the two highest resolution experiments. It is also apparent that cases 1408 and
1608 show uncharacteristic variations (when compared to the other truncations) beyond their first maximum.

To complete this overview on the behavior of our truncation experiments, Fig. 5 describes the time fluctuations of the zonal and eddy parts of both the total kinetic energy and the available potential energy throughout the integration period for each case. Since the predominant part (about 25 to 1 ) of the available potential energy is zonal, it is hardly astonishing that this energy has a time variation almost identical to the total available potential energy as seen from Fig. 4. The fluctuations in eddy available potential energy show little correspondence beyond day 50 and this only for the last five experiments, an anticipated feature for a parameter of such small relative magnitude.

The zonal KE, as shown in Fig. 5, shows a systematic tendency to reach its first and major maximum for later integration time with increasing resolution. Although this similarity of time dependence deteriorates with time, the energy level stabilizes to roughly similar values for the last four (highest resolution) experiments. Little more may be said for the eddy KE except that a tendency toward similarity of behavior with time is evident between the last two experiments.

Perhaps the most evident feature of Figs. 4 and 5 is 

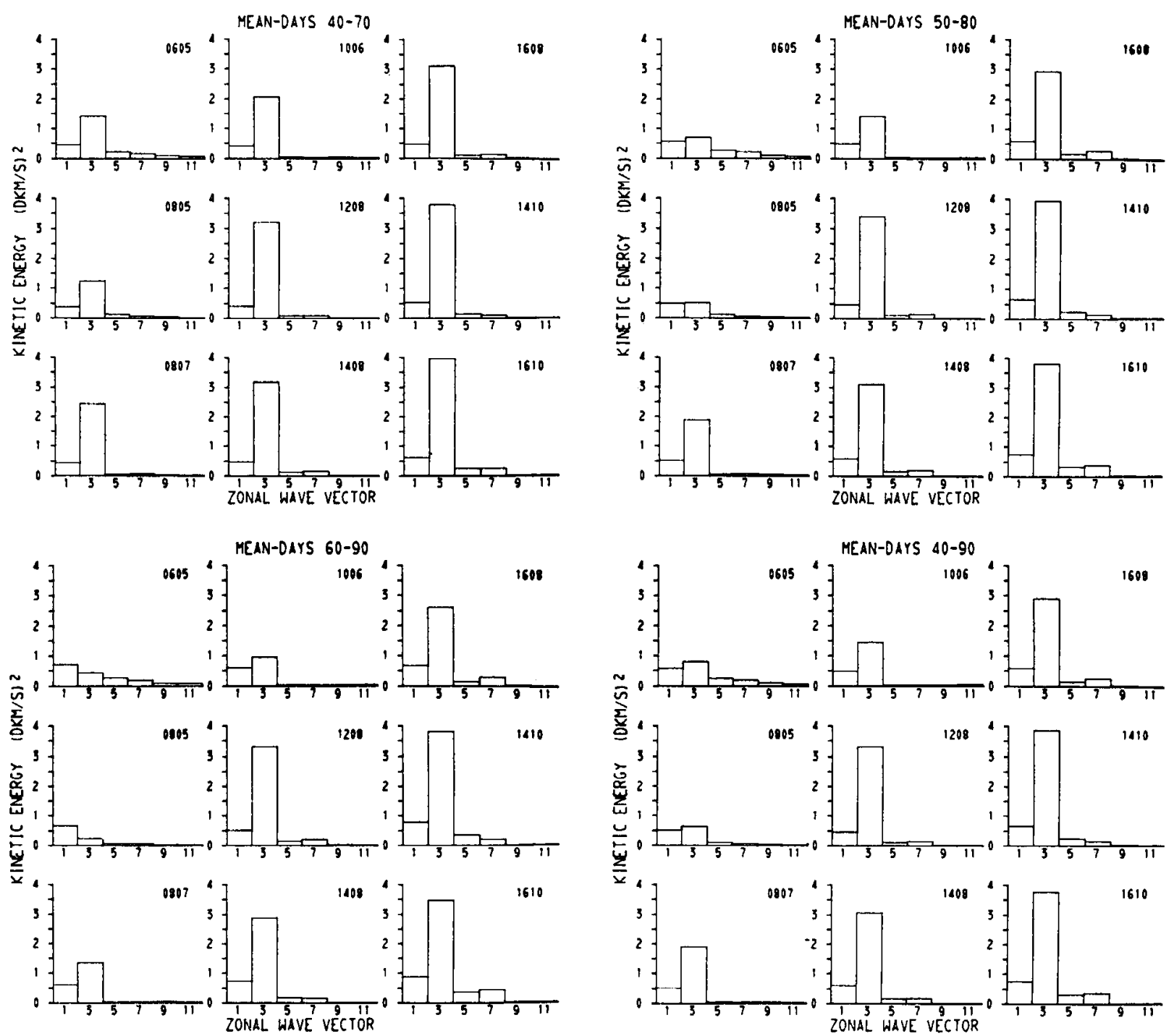

FıG. 7. Thirty-day mean zonal kinetic energy distributions $\left[\left(\mathrm{dkm} \mathrm{sec}^{-1}\right)^{2}\right]$ for zonal components $\gamma=1,3,5,7,9,11$, for all nine truncation experiments.

the increase in total energy in systems with greater resolution despite the fact that the forcing function is identical for all truncations and that the energy remains confined primarily to the basic subset as evidenced by Table 2. We shall attempt to uncover further effects of truncation by considering the properties summarized on Figs. 4 and 5 in greater detail.

\section{The general circulation}

We have seen the behavior in time of the gross energetics for the different experiments in Figs. 4 and 5. Although we note the lack of exact similarity of the different experiments toward the end of the integration period, we may yet find that some of the higher resolution cases yield similar solutions on the average, which is to say that they describe the general circulation for our model in a similar way. To establish the extent of this similarity, we have chosen to interpret 30-day running time means of the energy components and also one 50-day mean (from day 40 to day 90); hopefully, 30-day means have some correspondence to monthly means.

Let us consider first the distribution of the mean zonal wind with latitude. Fig. 6 depicts this distribution for two 30-day periods and for each experiment. One sees inmediately the similarity in profile for the last five experiments, whereas for those experiments with less than 12 planetary waves the profiles not only vary significantly, but also change from one time period to another. Those experiments including at least 14 planetary waves show remarkable similarity in their zonal wind profiles and do not change significantly from one time period (30-day average) to another. There appears to be a gradual increase in the zonal wind amplitude with increasing resolution, a feature already noted 

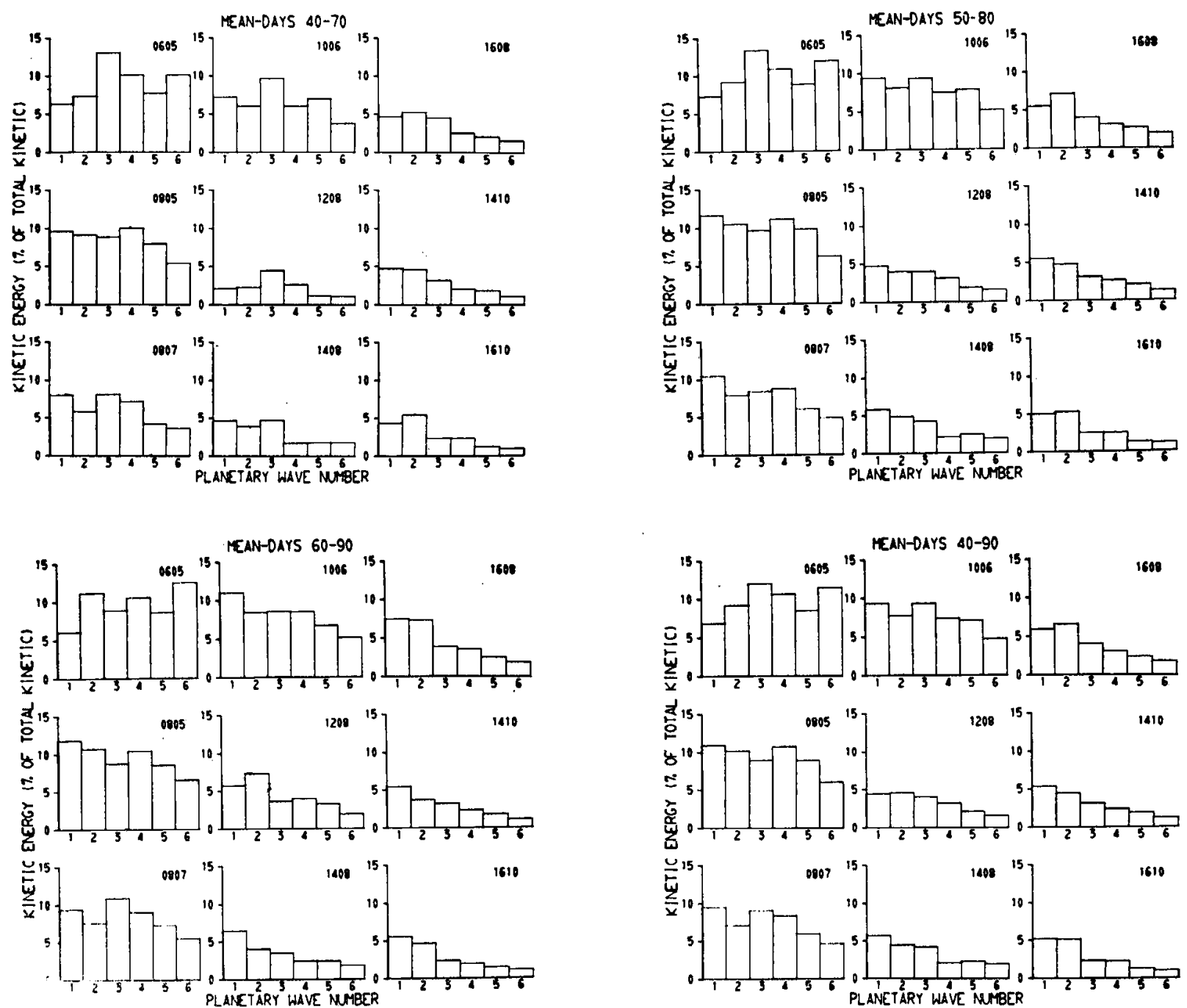

FIG. 8. Thirty-day mean eddy kinetic energy distributions in planetary waves $1-6$ given as percent of total kinetic energy for all truncation experiments.

from the increased energy in the zonal kinetic energy displayed on Fig. 5.

The zonal field as represented by Fig. 6 is made up of zonal spectral components which are completely specified by their energy because they have no imaginary part. Their prediction properties are of particular interest since they represent components which are forced by the heating function. We describe the kinetic energy in these components as averages for three 30-day integration periods and one 50-day period for all nine experiments by histograms on Fig. 7. It is apparent that a similarity in distribution among the components from case to case does not occur until at least 12 planetary waves are included, an observation which corresponds to the similarity noted in the zonal velocity field as represented in Fig. 6 . We also see that the vector $\gamma=3$ contains the bulk of the zonal kinetic energy in the five experiments of maximum resolution. Since the ratio established by the linear model as initial condi- tions at $t=36$ days is $E(\gamma=3) / E(\gamma=1) \approx 3$, the experiments with higher resolution not only maintain, but amplify this ratio. There is, furthermore, little change in the relative energy distribution among the wave components from one time period to another (this might also have been anticipated from Fig. 6) except for a gradual but not pronounced decrease in energy of wave $\gamma=3$ for case 1610. The last two experiments, 1410 and 1610, show particularly good correspondence in the amplitude of wave $\gamma=3$.

We have seen from (3.3) that the effects of heating go directly into changing the available potential energy. The component distribution of this energy in the zonal field is shown in Table 3. This table indicates that contrary to the kinetic energy distribution with its predominant maximum in $\gamma=3$, more potential energy is found in the first component $(\gamma=1)$; the remaining active components have several orders of magnitude less energy and are therefore not tabulated. Moreover, 

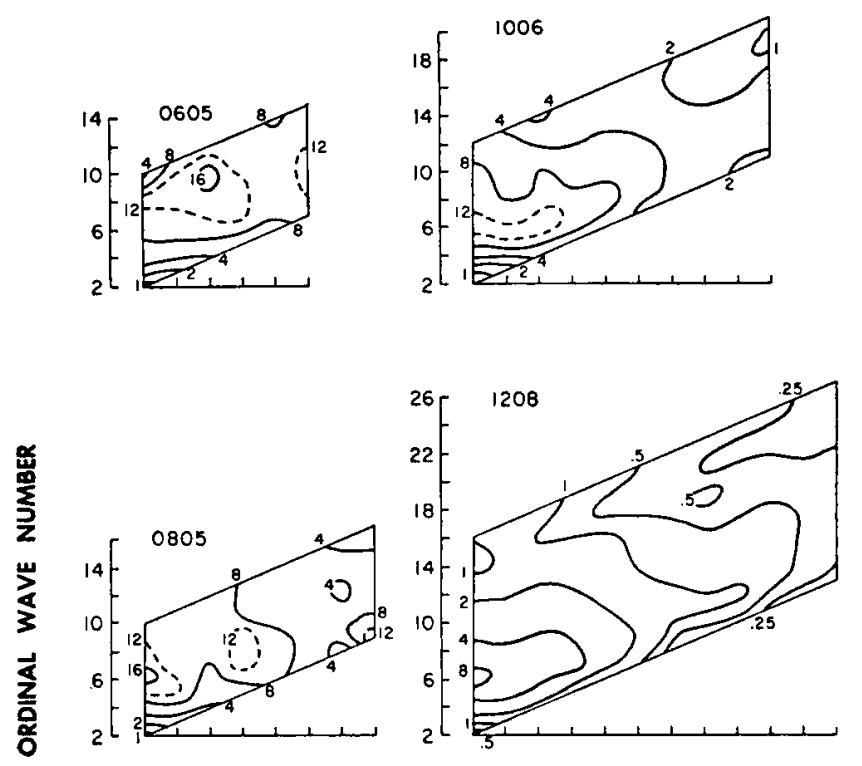

\section{MEAN - DAYS 4090}
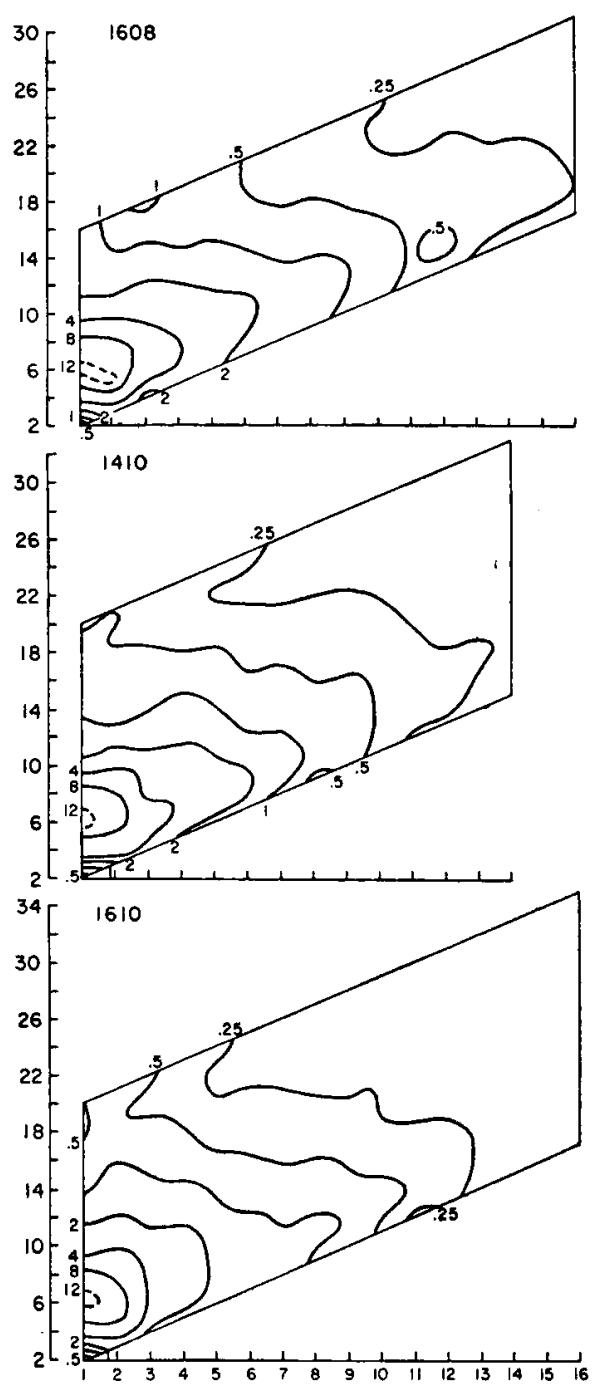

PLANETARY WAVE NUMBER

FIG. 9. Fifty-day mean of spectral kinetic energy- $\left[\left(\mathrm{m} \mathrm{sec}^{-1}\right)^{2}\right]$ distribution in all included wave vectors analyzed on $n-l$ diagrams for all nine truncation experiments (see Fï. 2).

this energy increases in time for $\gamma=1$ while decreasing for $\gamma=3$, although it increases for both components with increasing resolution. Thus, we see once again the significant changes in the basic subset (here the primary forced components) with increased resolution, indicat-

TABLE 3. Mean available potential energy $\left[\left(\mathrm{m} \mathrm{sec}^{-1}\right)^{2}\right]$ in zonal components $\gamma=1,3$ for selected periods for all experiments.

\begin{tabular}{rrrrrrrrrrrrr}
\hline $\begin{array}{l}\text { Wave } \\
\text { vector } \\
\gamma\end{array}$ & $\begin{array}{l}\text { Period } \\
\text { (days) }\end{array}$ & 0605 & 0805 & 0807 & \multicolumn{1}{c}{$\begin{array}{c}1006 \\
\text { d }\end{array}$} & 1208 & 1408 & 1608 & 1410 & 1610 \\
\hline 1 & $40-70$ & 606 & 584 & 640 & 605 & 603 & 699 & 699 & 725 & 830 \\
3 & $40-70$ & 249 & 244 & 428 & 34.3 & 663 & 551 & 541 & 622 & 586 \\
1 & $60-90$ & 962 & 926 & 859 & 857 & 732 & 1026 & 971 & 1039 & 1165 \\
3 & $60-90$ & 91 & 61 & 175 & 132 & 529 & 434 & 359 & 517 & 467 \\
\hline
\end{tabular}

ing some form of catalytic involvement by the additional degrees of freedom.

To assess the influence of truncation on the energy distribution in the different planetary waves, we have prepared histograms of the planetary wave kinetic energy as defined by (40) for several 30-day means in percent of the total kinetic energy and displayed them for each experiment in Fig. 8. Because of the preponderance of energy in the first six waves and because all experiments contain at least six waves, we have limited our analysis to these waves. We note immediately the obvious reduction of relative wave energy with increasing resolution, a feature which also holds true for the non-normalized energy. Furthermore, a common distribution among the waves appears only after at least 12 planetary waves are included in an 

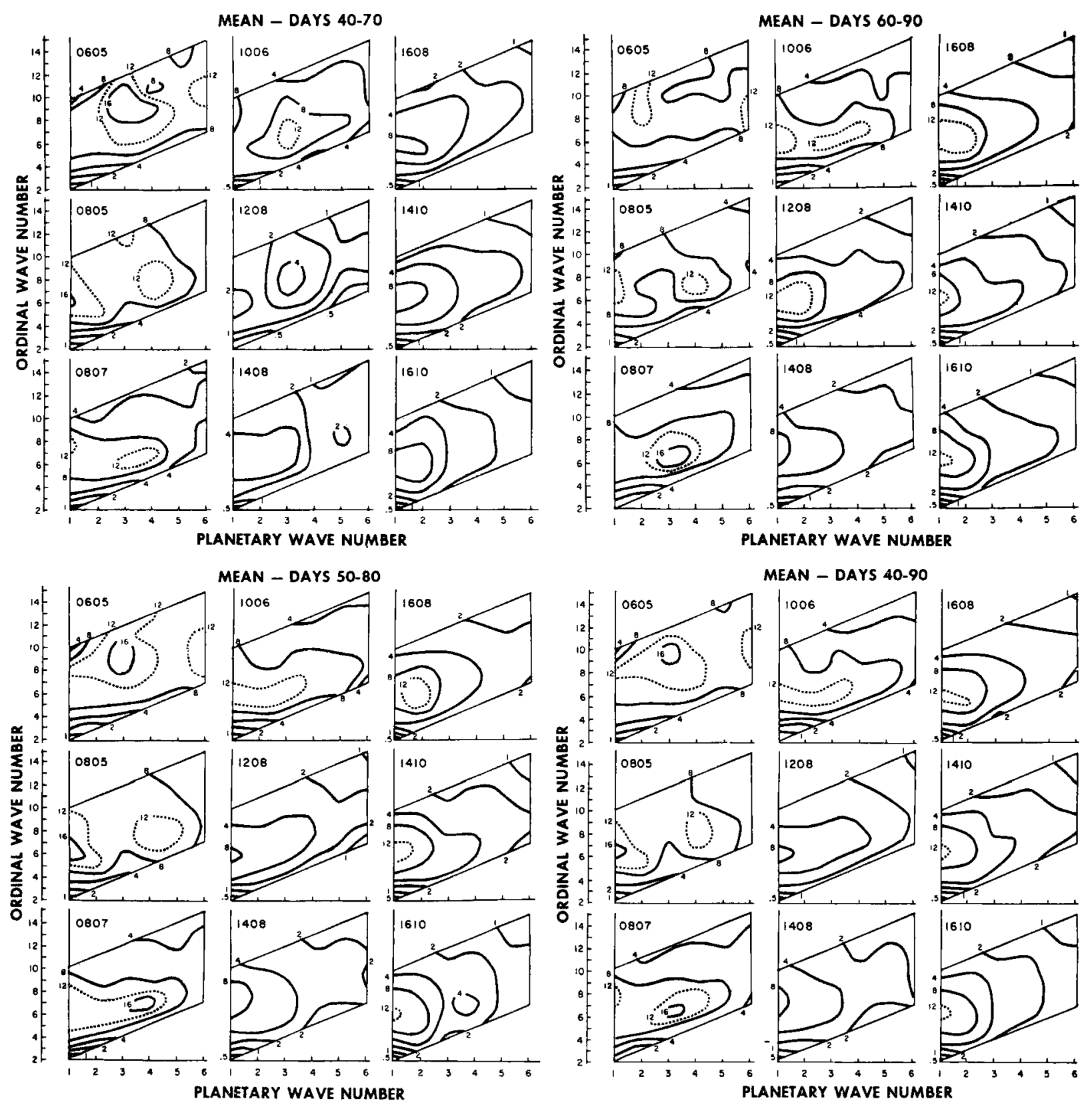

Fis. 10. Selected thirty-day mean spectral kinetic energy $\left[\left(\mathrm{m} \mathrm{sec}^{-1}\right)^{2}\right]$ distribution in the basic subset (0605) analyzed on $n-l$ diagrams for all nine truncation experiments.

experiment, thereby excluding the first four truncations which show a strong tendency toward erratic distributions. This similarity among wave distributions becomes yet more pronounced for the last three experiments, and their variation from one time period to another and to the overall time mean is not pronounced. For the highest resolution experiments, this similarity makes the relative influence of increasing $n$ or $l$ components difficult to assess.

Although Fig. 8 implies a tendency for similar energy clistributions among planetary waves for the higher resolution cases, the energy in each wave may not be distributed similarly with latitude. To determine these distributions, we have prepared spectral maps of kinetic energy in each $\gamma$ component for each experiment and for several 30-day periods. These maps were prepared by plotting the energy in each vector component at the corresponding location of its vector on an $n-l$ diagram (Fig. 2) and analyzing the energy field. Fig. 9 displays nine such maps (one for each experiment) for the average energy over the integration period of 40-90 days. This figure indicates that at least for the five highest resolution experiments the latitudinal distribution is not random but tends to be 
similar from one experiment to another with the patterns on the last three cases comparing quite acceptably. There is, however, a tendency toward reduced energy amplitude in the shorter waves with higher resolution, as suggested by Table 2. Since most of the energy is confined to the basic subset (0605) for all integrations, we have prepared Fig. 10 which compares only the energy in the basic subset for all experiments and for averages of several time periods; the analyses in the lower right of this figure are clearly the subset details of Fig. 9. We note immediately the strong tendency toward a maximum in the vicinity of $n \approx 6-8$ and as already seen from Fig. 8, a maximum for the largest planetary wave $(l=1)$. The tendency toward reduced energy in all components with increased resolution has already been demonstrated and needs no further comment. Comparison of cases 1408 with 1608 and 1410 with 1610 suggests that increasing the resolution in latitude is more influential in decreasing component energy than increasing the number of planetary waves with fixed latitudinal resolution. Nevertheless, the distributions of all four cases show surprising similarity. Fig. 10 also implies that pronounced variations in spectral energy distribution do not occur from one 30day mean period to another, particularly for the highest resolution integrations.

Although we have no evidence that integration with yet higher resolution would not yield different energy distributions from those we have observed, the tendency toward similarity among those experiments with at least twelve planetary waves as evidenced from Figs. 6-10 lend credence to the observation, at least for the model considered in this investigation, that a moderately successful description of the general circulation can be achieved with a truncation including at least twelve planetary waves and eight latitudinal degrees of freedom.

\section{Long-range prediction}

The ability of a model with given space truncation to satisfactorily describe properties of the general circulation does not necessarily imbue it with a similar capability for detailed time prediction of those properties. To assess this capability of our model as a function of truncation, we describe some of the parameters discussed in the previous section in terms of their detailed time behavior for the first 25 days of integration (days $36-60)$ for all nine experiments.

For the gross energetics of the model we may refer to Fig. 4, confining our attention to the first 25 days. The total energy shows very sinilar time behavior for the first 15 days among the last five experiments after which truncations 1408 and especially 1608 show a pronounced leveling-off tendency from energy growth. A similar conclusion may be drawn from the development of the available potential energy, although fluctuations from steady growth begin to appear. These fluctuations become considerably more pronounced in the total kinetic energy where significant deviations from similarity begin to appear beyond 15 days except for experiments 1410 and 1610, which behave identically (in this parameter) for almost 25 days. A notable exception is the performance of case 1608 ; since there is no systematic reason for this erratic behavior, we must acknowledge the possibility of computer error.

For the detailed variation in the zonal and eddy available potential energy, let us refer to the first 25 days on Fig. 5. Because of its relative amplitude, the zonal APE behaves much as the total APE discussed above. The eddy APE begins to show significant variation after 10 days of integration in the last five experiments with only truncations 1410 and 1610 following one another closely for over 20 days. (ase 1608 again displays uncharacteristic behavior.

Because of their importance in describing the flow field, we have displayed the zonal $\mathrm{KE}$ and eddy $\mathrm{KE}$ on an expanded time scale (35-60 days) in Fig. 11. In terms of nondimensional units, the zonal $\mathrm{KE}$ time variation is described similarly for about 10 days by the last five experiments, after which significant deviations appear in all but truncations 1410 and 1610 . A similar observation may be made for the eddy $\mathrm{KE}$, despite the fact that the second relative maximum does appear in all experiments, but unfortunately at different integration times. Although of little consequence to the prediction of the general circulation the latter disparity could undoubtedly lead to significant differences in the long-range prediction results of the various cases. Included on Fig. 11 is the time distribution of zonal and eddy $\mathrm{KE}$ as normalized to the total $\mathrm{KE}$. Comparison of the nondimensional to normalized curves shows that over the 25-day integration period the zonal $\mathrm{KE}$ increases but the relative energy in the zonal field decreases, implying that the waves are receiving relatively more energy than the zonal flow; all cases reproduce this feature, but not with comparative similarity except again for cases 1410 and 1610 .

The detailed structure of the zonal field may be seen from the behavior of components $\gamma=1,3,5$ and their sum. Their nondimensional kinetic energy time variations for the first 25 days are given in Fig. 12. Although the fluctuations in component $\gamma=1$ are extremely smooth and compare favorably for all models, the component of primary significance and which accounts for most of the total zonal amplitude is $\gamma=3$ (this has already been established from Fig. 7). We again note that satisfactory comparative behavior of the last five experiments ends after 15 days except for 1410 and 1610, which continue to correspond for over 20 days. The pronounced energy decrease in the lower resolution experiments as compared to an increase in the higher resolution ones emphasizes our earlier observation of the dramatic difference in solution as a function of truncation, especially since $\gamma=3$ is one of the primary forced components. 

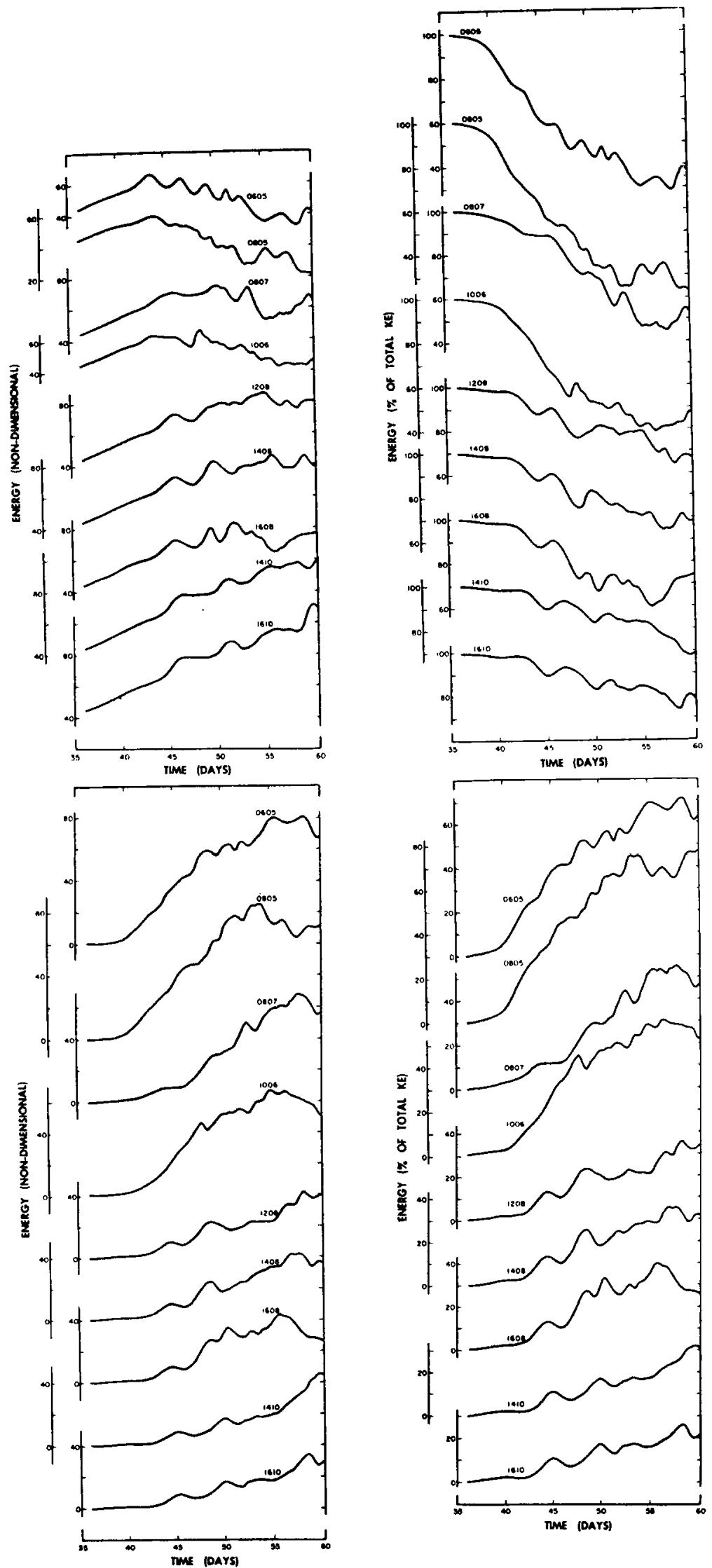

lifg. 11. Zonal (top) and eddy (bottom) kineticenergy in both nondimensional units and as a percent of total kinetic energy as a function of time to day 60 for all nine truncation experiments. 

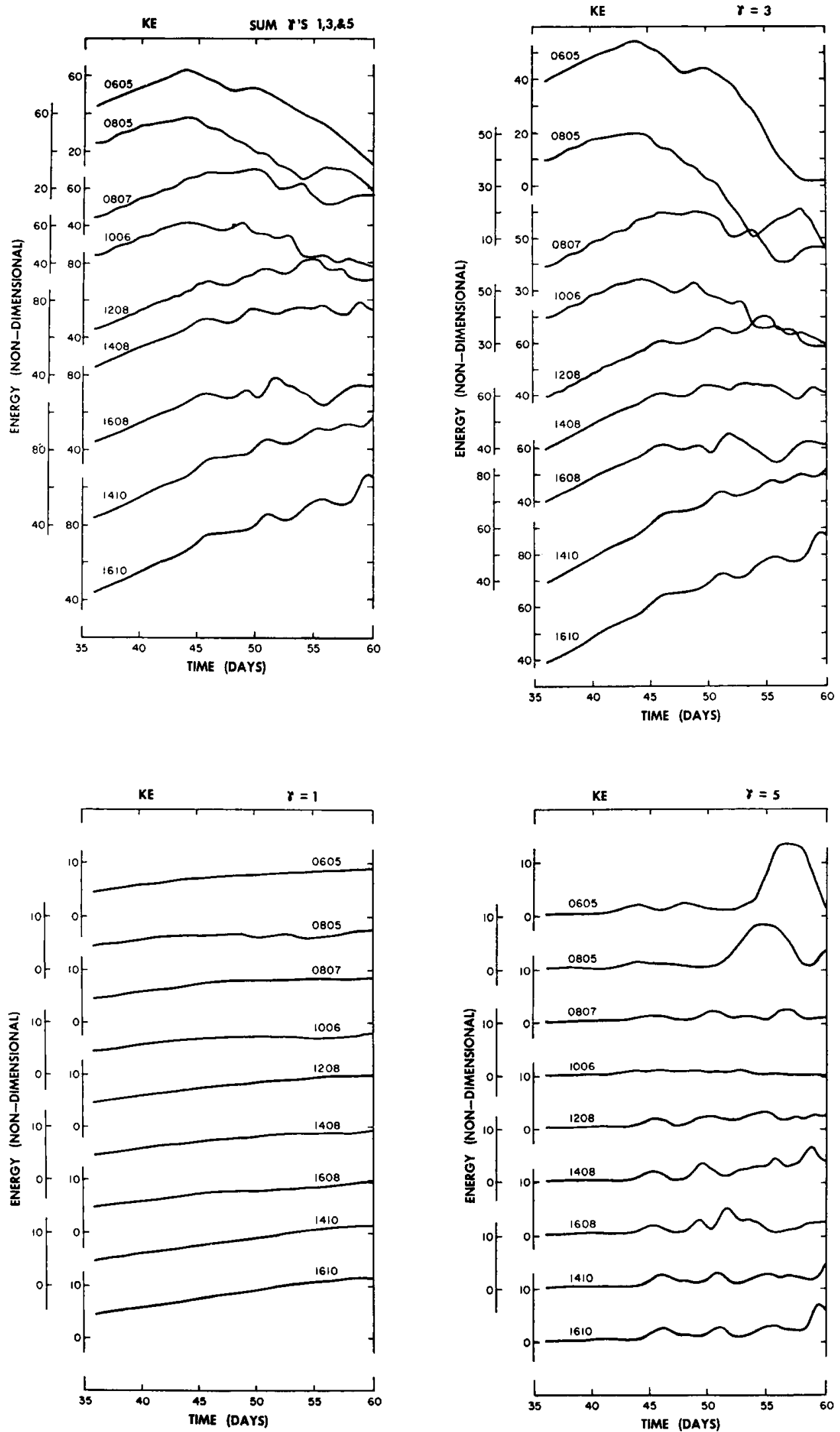

Fic. 12. Kinetic energy in zonal components $\gamma=1,3,5$ and their sum in nondimensional units as a function of time to day 60 for all nine truncation experiments. 

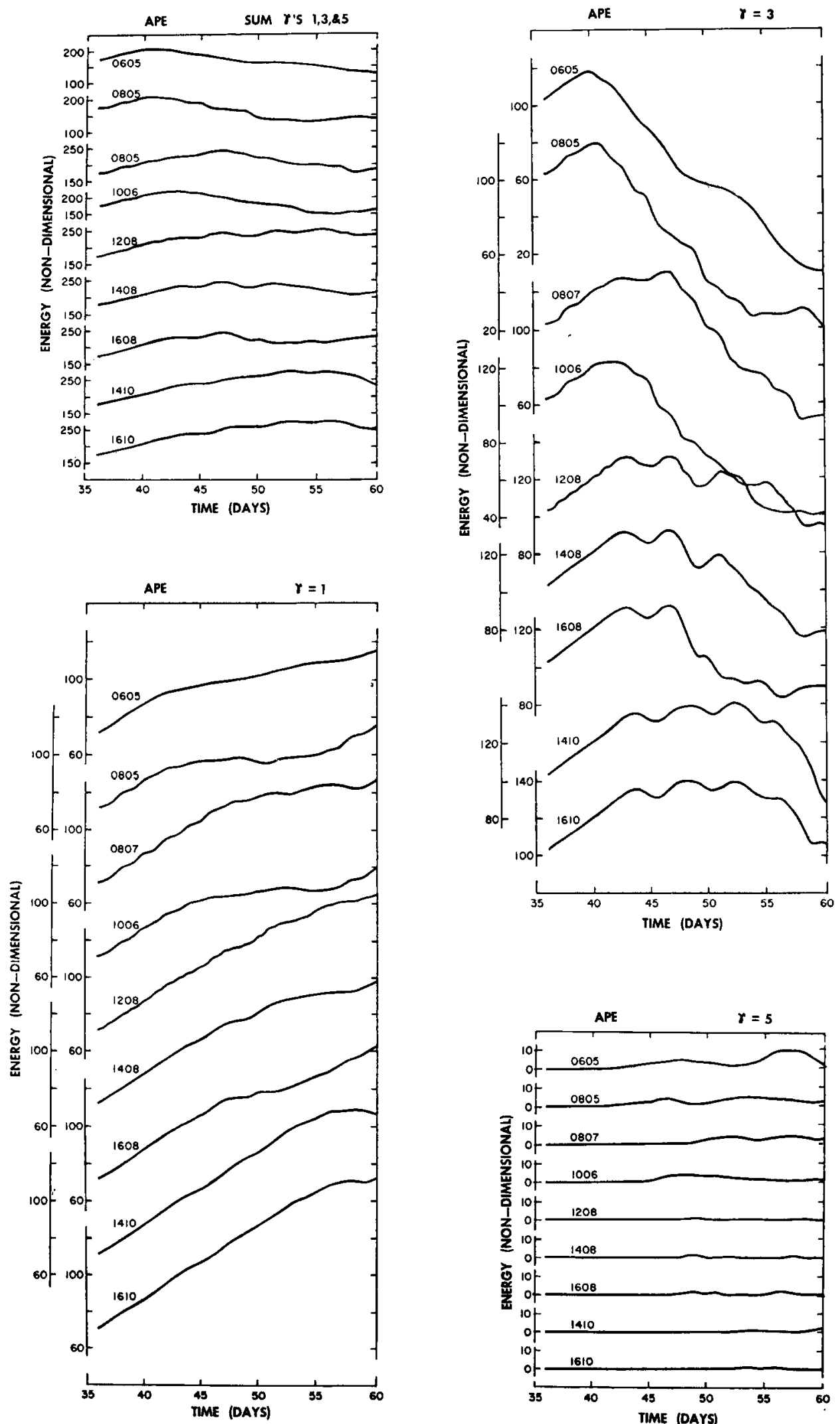

Fig. 13. Availalsle potential energy in zonal components $\gamma=1,3,5$ and their sum in nondimensional units as a function of time to day 60 for all nine truncation experiments. 

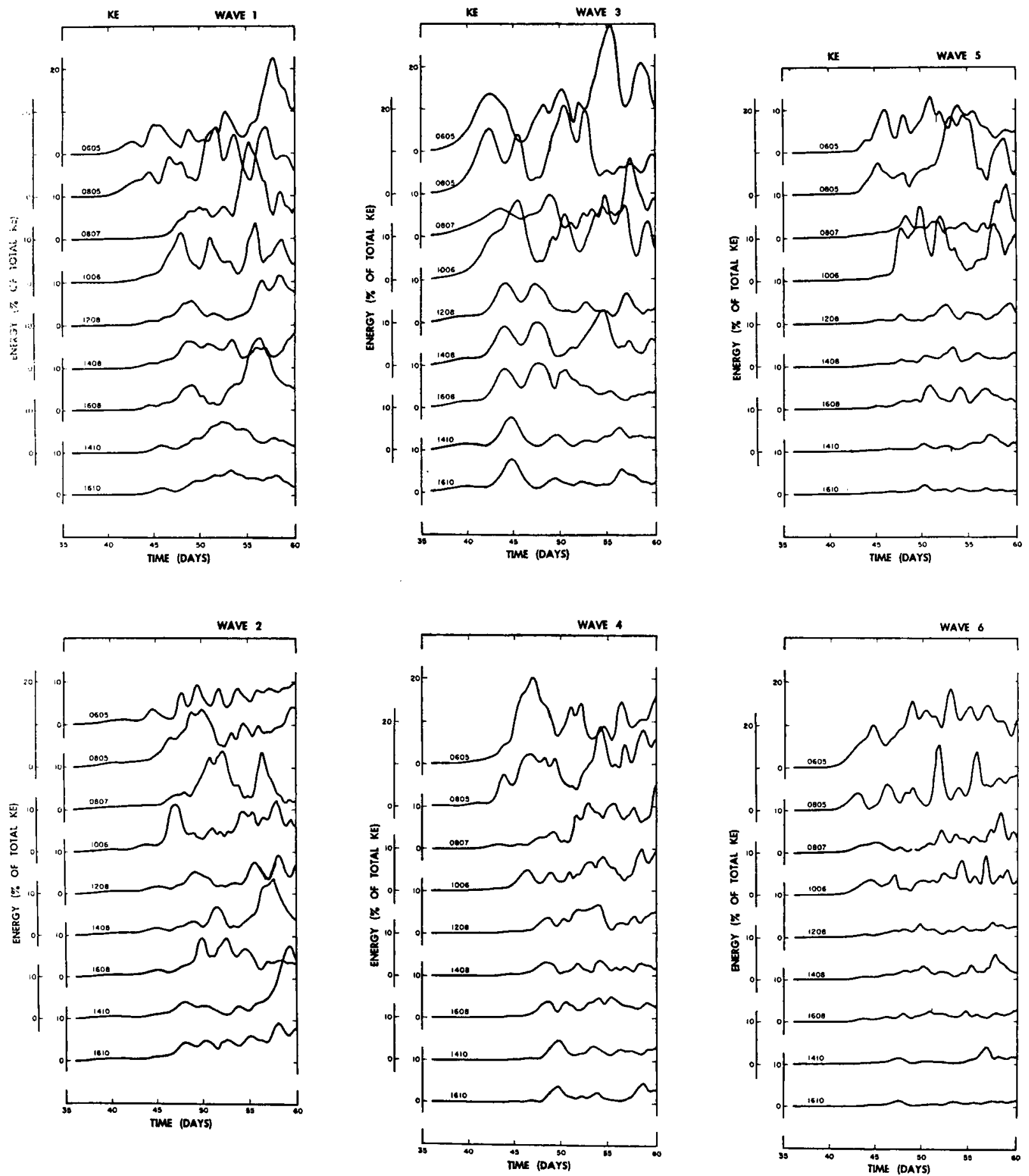

FIG. 14. Kinetic energy in planetary waves $1-6$ as a percent of total kinetic energy as a function of time to clay 60 for all nine truncation experiments.

Fig. 1.3 depicts the variation of the available potential energy for the same zonal components shown in Fig. 12. The conclusions for potential energy are similar to those discussed above for kinetic energy, but we note that growth of the $\gamma=1$ component is quite pronounced and toward the end of the 25-day period has greater amplitude than the $\gamma=3$ component. Again we see that only cases 1410 and 1610 correspond closcly beyond about 10 days of integration, and that truncation 1608 shows comparatively erratic behavior.

Finally, we describe the time dependence of the total kinetic energy in each of the first six planetary waves, effectively a detailed breakdown of the eddy $\mathrm{KE}$ curves shown in Fig. 11. These variations have been plotted 
on Fig. 14 in terms of percent of total KE for all nine experiments. Confining our attention again to only the last five experiments, it is apparent that significant growth of energy in any of these six waves does not occur for the first ten days, which may account for the satisfactory comparative behavior in this time range of the cumulative properties already discussed. However, beyond ten days the integrations of cases 1410 and 1610 compare quite favorably for at least another ten days, whereas the other experiments show pronounced variations one from the other.

\section{Conclusions}

In order to establish the dependence of numerical nonlinear integrations of an atmospheric model on space truncation, a two-level, quasi-geostrophic, fixed stability model with heating, momentum diffusion, and internal and external stress was integrated in time for at least 95 days for nine different truncations. The model was represented in spectral form and the truncation was established spectrally rather than in the point domain. To avoid confusion with regard to initial conditions, all truncations were initiated with the forced zonal distribution generated from rest at which wave activity (nonlinearity) first appeared.

The analysis of the integration results were presented separately for the general circulation and for longrange prediction since the former deals with timeaveraged properties while the latter requires exposition of exact time variations. IDiscussion of a system's behavior was confined to the energetics save for a brief display of the zonal flow and its latitudinal dependence. Comparisons were made of the integrations with all nine truncations experiments ranging from six to sixteen planetary waves and from five to ten degrees of latitudinal freedom. Perhaps the most pronounced feature of the calculation was the lack of increase in shortwave activity with increasing resolution; this feature was also observed by Manabe et al. (1970), although they showed pronounced increase in the energy level of the longer waves with increased resolution, clearly not seen in our experiment. Although solutions for different truncations varied broadly, little activity was seen (in any experiment) outside of the wave regime defined by the lowest resolution experiment. We are thus led to the conclusion that the additional degrees of freedom added by increasing resolution are not an energy drain but act rather in a catalytic fashion to redistribute energy among the larger wave component.

Twelve planetary waves are a mininum number which must be included in our model to give a reasonable description of the general circulation, although fourteen or even sixteen would be preferable. Concurrently, at least eight degrees of freedom in latitude should be included. One must interpret this result with some caution, however. Our model is forced only in the lowest zonal components; should one inclucle forcing in shorter waves as exemplified by latent heat release, the above

conclusion on truncation for a general circulation model could well be invalidated.

Regarding long-range prediction, our integrations suggest that for adequate prediction to 20 days at least. sixteen planetary waves are required with a minimum or eight latitudinal degrees of freedom. This limit is unfortunately at the maximum resolution range of our experiments, and calculations with more degrees of freedom could show further variations in the solution over the first 20 days. Nevertheless, the last two experiments (highest resolution) are satisfactorily comparable over this integration period. Because the wave activity at the initiation of our integration is infinitesimal, phase considerations are not of great consequence for some days into the integration. The results of the calculations are nevertheless pertinent since we must assume a "rrue" solution toward which the solution to higher resolution models tend. Were our model to be used with observed finite-amplitude initial data, however, we have no assurance that the resolution requirements stated above would be adequate to yield satisfactory integrations for as long as 20 days.

The broad variations in integration results as dependent on space (spectral) truncation noted in this paper imply the need for similar experiments with models containing different forcing, especially if that forcing covers a broad wave range.

Acknowledgments. This research has been supported by the Atmospheric Sciences Section, National Science Foundation, under Grant GA-11637 to Colorado State University. Computer time and assistance has been donated by the Geophysical Fluid Dynamics Laboratory, Princeton, and the National Center for Atmospheric Research, Boulder.

\section{API'ENDIX}

\section{Model Data}

1. Stress, diffusion and stability constants

$$
\begin{aligned}
& c_{1}=0.5 \times 10^{-6} \mathrm{sec}^{-1} \\
& c_{2}=2 \times 10^{-6} \mathrm{sec}^{-1} \\
& .1=10^{-5} \mathrm{~m}^{2} \mathrm{sec}^{-1} \\
& \delta^{2}=3 \times 10^{-12} \mathrm{~m}^{-2} \\
& f_{11}=10^{-4} \mathrm{sec}^{-1}
\end{aligned}
$$

\section{Heating coefficients}

$$
\begin{aligned}
& c_{p} h_{2}=-168.95 \mathrm{ly} \mathrm{day}{ }^{-1} \\
& c_{p} h_{1}=-14.02 \mathrm{ly} \mathrm{day}^{-1}
\end{aligned}
$$

\section{Initial data (day 36 in nondimensional units)}

$\gamma$

1

3

5

7

9

11

$\begin{array}{cc}\psi & \tau \\ -0.91435 \times 10^{-2} & -0.54225 \times 10^{-2} \\ -0.11037 \times 10^{-1} & -0.65010 \times 10^{-2} \\ -0.58016 \times 10^{-3} & -0.33502 \times 10^{-3} \\ 0.57510^{-6} & 0.5025 \times 10^{-5} \\ -0.1 \times 10^{-6} & 0.12 \times 10^{-5} \\ -0.5 \times 10^{-6} & -0.16 \times 10^{-5}\end{array}$


For all $\gamma, 1 \leqslant l_{\gamma} \leqslant 6$ and $n_{\gamma}=l_{\gamma}-1+2 j(1 \leqslant j \leqslant 5)$

$$
\psi_{\gamma}(\text { real }) \approx 10^{-5} \quad \tau_{\gamma}(\text { real }) \approx 10^{-5}
$$$$
\psi_{\gamma}(\text { imag. }) \approx 10^{-5} \quad \tau_{\gamma}(\text { imag. }) \approx 10^{-5}
$$

\section{REFERENCES}

Baer, F., 1964: Integration with the spectral vorticity equation. J. Almos. Sci., 21, 260-276.

, 1968: Studies in low-order spectral systems. Paper No. 129, Dept. Atmospheric Science, Colorado State University, $77 \mathrm{pp}$.

--, and R. L. King, 1967: A general computational form for a class of nonlinear systems incorporating both spectral and finite-difference approximations. J. Comput. Pliys., 2, 32-60.

-, and G. W. Platzman, 1961: A procedure for numerical integration of the spectral vorticity equation. J. Meteor., 18, 393-401.

_- , and T. J. Simons, 1970: Computational stability and time truncation of coupled nonlinear equations with exact solutions. Mon. Wea. Rev., 98, 665-679.

Charney, J. G., 1959: On the theory of the general circulation of the atmosphere. The Atmosphere and the Sea in Motion, New York, The Rockefeller Institute Press, 178 193.

Davis, P. A., 1963: An analysis of the atmospheric heat budget. J. Atmos. Sci., 20, 5-22.

Elsaesser, H. W., 1966: Evaluation of spectral versus grid methods of hemispheric numerical weather prediction. $J . A p p l$. Meteor., 5, 246-262.

Hobson, E. W., 1955: The Theory of Spherical and Lillipsoidal Harmonics. New York, Chelsea, $500 \mathrm{pp}$.

Kasahara, A., and M. W. Washington, 1967: NCAR global general circulation model of the atmosphere. Mon. Wea. Rev., 95, 389-402.

Kikuchi, Y., 1969: Numerical simulation of the blocking process. J. Meteor. Soc. Japan, 47, 29-54.

Leith, C. E., 1965: Numerical simulation of the earth's atmosphere. Methods of Computational Physics, New York, Academic Press, 1--28.
Lettau, H. H., 1954: A study of the mass, momentum, and energy budget of the atmosphere. Arch. Meteor. Geopliys. Bioklim., A7, 133-157.

Lorenz, E. N., 1960: Energy and numerical weather prediction. Tellus, 12, 364-373.

- 1967 : The Nature and Theory of the General Circulation of the Atmosphere. Geneva, World Meteorological Organization, $161 \mathrm{pp}$.

- 1969 : The predictability of a flow which possesses many scales of motion. Tellus, 21, 289-307.

Manabe, S., J. Smagorinsky, J. L. Holloway, Jr. and H. M Stone, 1970: Simulated climatology of a general circulation model with a hydrolic cycle. III. Effects of increased horizontal computational resolution. Mon. Wea. Rev., 98, 175-212.

Merilees, P. E., 1968: The equations of motion in spectral form. J. Atmos. Sci., 25, 736-743.

Mintz, Y., 1958: Design of some numerical general circulation experiments. Bull. Res. Council Israel, 7G, 67-114.

-.., 1965: 'The general circulation and long-range prediction. Advances in Numerical Weather Prediction, Hartford, The Travelers Research Center, Inc., 6-23.

Phillips, N. A., 1956: The general circulation of the atmosphere: A numerical experiment. Quart. J. Roy. Meteor. Soc., 82, 123-164.

P'latzman, G. W., 1960: The spectral form of the vorticity equation. J. Meleor., 17, 635-644.

Richtmyer, R. D., 1957: Difference Melhods for Initial Value Problems. New York, Interscience $238 \mathrm{pp}$.

Robert, A. J., 1966: 'The integration of a low order spectral form of the primitive meteorological equations. J. Meteor. Soc. Japan, 44, 237-245.

Saltzman, B., 1958: Some hemispheric spectral statistics. $J$. Meteor., 15, 259-263.

Sellers, W. D., 1965: Physical Climatology. University of Chicago Press, $272 \mathrm{pp}$.

Smagorinsky, J., S. Manabe and J. L. Holloway, Jr., 1965: Numerical results from a nine-level general circulation molel. Mon. Wea. Rev., 93, 727-708.

Wiin-Nielsen, A,, 1967: On the annual variation of spectral distribution of atmospheric energy. Tellus, 19, 540 559 . 